Ref.TH.3692-CERN

\title{
DISCRETE SYMMETRIES IN KALUZA-KLEIN THEORIES
}

C. Wetterich

CERN -- Geneva

\section{ABSTRACT}

We investigate discrete symmetries in theories of higher dimensional (d > 4) gravity and their consequences for the reduced four-dimensional theory, obtained for a ground state which is a direct product of fourdimensional Minkowski space and a compact d-4 dimensional internal space. If the action of pure d dimensional gravity coupled to spinors is invariant under time reversal or reflection of an odd number of spacelike co-ordinates, the reduced four-dimensional theory has a non-trivial parity or CT symetry not consistent with observation. A non-trivial d dimensional charge conjugation results in an unwanted doubling of the four-dimensional fermion spectrum. As a consequence, realistic theories can only be obtained for Majorana-Weyl spinors in $\mathrm{d}=2 \bmod 8$ dimensions. The constraints are less stringent if supplementary fields are introduced in d dimensions. For $d=11$ supergravity, for example, parity and $C T$ invariance $c a n$ be broken by a non-vanishing field strength of the totally antisymetric
three-index tensor.

A ground state invaxiant under reflections of "internal" co-ordinates often gives rise to a non-trivial charge conjugation in four dimensions. We find that the ground state of a realistic Kaluza-Klein theory should (which cannotiant under any non-trivial internal co-ordinate reflection on a possiblet be obtained by a gauge transformation). We finally comment and discuss solution of the strong CP problem from Kaluza-Klein theories fermions. 


\section{INTRODUCTION}

An explanation why nature violates parity and time reversal, or equivalently, $\mathrm{CP}$ conjugation, is one of the old puzzles in particle physics. In four dimensions, pure gravity coupled to matter fields (without gauge interactions) is not only invariant under the continuous symmetry of global Poincare transformations (this symmetry is left invariant by Minkowski space), but also with respect to a reflection of an odd number of spacelike coordinates (parity) and time (time reversal). Paxity and time reversal are discrete symmetries which cannot be obtained from unity by an integration of infinitesmal Poincaré transformations.

Electromagnetic and strong interactions conserve $P$ (parity) and $T$ (time reversa1). The discovery of parity violation in weak interaction phenomena, and subsequently the discovery of $\mathrm{CP}$ violation which is equivalent to $\mathrm{T}$ violation by the CPT theorem, was one of the revolutions in particle physics. Up to now, our answers why $P$ and $C P$ are violated are only partly satisfactory. In some unified gauge theories based on SU(5) P-asymmetry can be linked to the existence of chiral fermions. This is only partly true in theories admitting a non-trivial left-right symmetry like SO(10). In this case, chiral fermions are not a sufficient condition for parity violation. No reason why $\mathrm{CP}$ has to be violated is provided by unified gauge theories, except phenomenological ones like our existence which requires a $\mathrm{CP}$ violating production of a baryon asymmetry in the early universe.

Kaluza-Klein theories of higher dimensional gravity are devised to unify gauge intexactions and gravity ${ }^{1)}$. One may hope that a natural explanation of the origin of $\mathrm{P}$ and $\mathrm{CP}$ violation could arise from this unification. Moreover, the existence of a four-dimensional charge conjugation is related to coordinate reflections in the supplementary dimensions and acquires a simple geometrical interpretation ${ }^{2}$. In this paper we investigate discrete symmetries in higher dimensional theories and their consequences for the reduced four-dimensional theory. We study conditions on the higher dimensional theory which are necessary for a violation of parity, charge conjugation, time reversal, and $\mathrm{CP}$ in the reduced theory. We find that these conditions are far from being trivial. Almost all the simple 
Kaluza-Klein models discussed so far have non-trivia1 P, C, or CP symmetries in the reduced four-dimensional theory.

In Section 2 we first derive the formalism to describe $C, P$, and $T$ in an arbitrary number of dimensions. In contradistinction to four dimensions, pure gravity coupled to matter has not necessarily $\mathrm{P}$ or $\mathrm{CP}$ and $\mathrm{T}$ invariance. Whereas Weyl spinors coupled to gravity in $d=4 \bmod 4$ violate parity, but conserve $C P$ and $T$, Weyl spinors in $\mathrm{d}=2 \bmod 4$ violate $\mathrm{P}$ and $\mathrm{T}$ whereas $\mathrm{C}$ and $\mathrm{PT}$ are conserved. This difference is related to the fact that for $d=4 \bmod 4$ Weyl spinors belong to complex representations of the Lorentz group, whereas for $d=2$ mod 4 they are in real or pseudoreal representations. For Majorana spinors, d-dimensional charge conjugation is trivial. As a consequence, the only non-trivial discrete symmetry of a Majorana-Weyl spinor in $d=2 \bmod 8$ is PI symmetry, which is the equivalent of CPT for Majorana spinors. Neither $\mathrm{P}$ nor $\mathrm{T}$ separately are symmetries of $\mathrm{a}$ theory of pure gravity coupled to a Majorana-Wey1 spinor. This could be the fundamental origin of the observed $P$ and $C P$ violation in nature.

In Section 3 we discuss the consequences of the symmetries $\mathrm{T}_{\mathrm{d}}$ ( $\mathrm{d}$-dimensional time reversal), $P_{3}$ (d-dimensional reflection of the first three space-like coordinates) and $e_{d}$ (d-dimensional charge conjugation) for the reduced four-dimensional theory in detail. In pure gravity theories coupled to spinors the ground state is defined by the vacuum value of the metric (or the vielbein) and we assume that the ground state is a direct product of four-dimensional Minkowski space $M^{4}$ and some $\mathrm{D}=\mathrm{d}-4$ dimensional "internal" space $\mathrm{K}^{\mathrm{D}}$. Such a ground state does not break $\mathrm{T}_{\mathrm{d}}$, $P_{3}$ or $\mathcal{C}_{\mathrm{d}}$ and these symetries reflect themselves as Four-dimensional discrete symmetries. $\mathrm{P}_{3}$ leads to some kind of four-dimensional parity, $\mathrm{T}_{\mathrm{d}}$ induces fourdimensional CT transformations (not $T$ transformations) and a non-txivial $\mathcal{C}_{d}$ generates an unobserved discrete symetry between pairs of fermions belonging to the same representation and with the same mass. None of these symmetries is conserved in four dimensions. As a consequence, realistic Kaluza-Klein theories of pure gravity coupled to a spinor should not admit $\mathrm{P}_{3}, \mathrm{~T}_{\mathrm{d}}$, or a non-trivial $\mathrm{e}_{\mathrm{d}}$ invariance: They 
must involve Majorana-Weyl spinors and this is only possible in $\mathrm{d}=2 \bmod 8$ dimensions. It is interesting to note that this is the only case consistent with the existence of chiral spinors ${ }^{3}$ and just what is needed to explain the origin of the breaking of discrete symmetries!

In Section 4 we discuss reflections of the coordinates parametrizing the internal space $\mathrm{K}^{\mathrm{D}}$. Four-dimensional charge conjugation can be described by such reflections. If $\mathrm{K}^{\mathrm{D}}$ is invariant under some coordinate reflection, this reflection corresponds to an automorphism of the algebra of the gauge group. Some abelian charges will change their sign. If the reflection can be obtained by a gauge transformation we have an inner automorphism which is harmless. In the other case, we have non-trivial internal discrete symetries corresponding to an outer automorphism. Reflections of an odd number of coordinates are always non-trivial. The outer automorphism can either carry every complex representation in the harmonic expansion on $\mathrm{K}^{\mathrm{D}}$ in its complex conjugate one. Then it corresponds to a non-trivial charge conjugation in four dimensions. E1se, the automorphism transforms complex representations into equivalent, but different representations and results in a doubling of degrees of freedom in four dimensions (two different electrons with the same mass, etc.). None of these discrete symmetries is observed in nature. This implies that the ground state of a realistic Kaluza-Klein theory of pure gravity should not admit any non-trivial discrete symmetries.

The existence of non-trivial discrete symmetries is also very dangerous for the possible existence of chiral fermions on such spaces. In many cases specific discrete symmetries of $k^{D}$ are sufficient to rule out chiral spinors in the fourdimensional spectrum. This becomes a powerful constraint on realistic KaluzaKlein theories when combined with the fact that the number of chiral fermions is related to topological properties of $\mathrm{K}^{\mathrm{D}}{ }^{4}$ ).

In Section 5 we show how the constraints on realistic Kaluza-Klein theories derived from an investigation of discrete symmetries can be released if one introduces supplementary matter fields in higher dimensions. This is especially relevant 
for supergravity theories. Supergravity in eleven dimensions is known to be $T_{d}$ invariant ${ }^{5}$ and, as a consequence of the higher dimensional generalization of the CPT theorem, has also a non-trivial invariance with respect to an odd number of coordinate reflections, in particular $\mathrm{P}_{3}$. Nevertheless, four-dimensional parity can be broken due to a non-vanishing vacuum value of the field strength of the totally antisymetric three index tensor ${ }^{6}$ ).

In the last section we discuss two other problems in the light of our results. The first topic concerns the problem of strong CP violation by a term $\sim \theta F_{\mu \nu} \tilde{F}$ in the effective four-dimensional action. We argue that it is very likely that Kaluza-Klein theories provide a solution to this problem. If pure higher dimensional gravity is $P_{3}$ invariant and $P_{3}$ violation comes only from the fexmionic sector, no term $\sim \theta \mathrm{F}{ }_{\mu \nu} \tilde{F}^{\mu \nu}$ can be induced by dimensional reduction since the ground state is invariant under $P_{3}$. Thus the $\theta$ parameter vanishes at the compactification scale and only small calculable corrections are induced by fermion loops.

The second topic concerns some requirements on the local form of the metric of a space $\mathrm{K}^{\mathrm{D}}$ admitting chiral fermions. We find that the possibility of obtaining unwanted discrete symmetries by continuous deformations of the internal space implies severe difficulties for finding ground states admitting chiral fermions.

\section{2. $\mathrm{C}, \mathrm{P}, \mathrm{T}$ AND ALL THAT IN HIGHER DIMENSIONS}

We start with a discussion of discrete symmetries in higher dimensions. In this section we concentrate on models of pure gravity in d dimensions coupled to a d dimensional spinor field. Models with supplementary matter fields (like $\mathrm{d}=11$ supergravity) will be discussed in Section 5 . The action of these models is invariant under general coordinate transformations in d dimensions and undex d-dimensional Lorentz rotations which form the local group so(d - 1, 1) [or, for spinors, the corresponding Spin $(d-1,1)$ group]. We call this combined symmetry group gen $\mathrm{d}$. 
The action is of the form

$$
S=-\int d^{d} \times \hat{g}^{1 / 2}\left(f\left(\hat{g}_{\mu \nu}\right)-i \bar{\psi} \gamma^{\hat{m}} \hat{e}_{\dot{m}}^{\hat{\mu}} D_{\hat{\mu}} \psi\right)
$$

[For investigations of spontaneous compactification it is appropriate to consider (1) as the effective action ${ }^{7)}$ at the length scale characteristic for the internal space. Such an effective action includes quantum fluctuations.] Here $\psi$ is a spinorial representation of Spin (d - 1, 1) with anticommuting properties $\psi_{\alpha} \psi_{\beta}=-\psi_{\beta} \psi_{\alpha}$, $\hat{e}_{\hat{\alpha}}^{\hat{m}}$ is the d-dimensional vielbein, $\hat{e}_{\hat{\mathrm{m}}}^{\hat{\rho}}$ the inverse vielbein and $\hat{g}_{\hat{\mu} \mathcal{O}}=e_{\hat{\mu}}^{\hat{m}} e_{\hat{\nu}}^{\hat{n}} \eta_{\hat{m} \hat{n}}$ the corresponding metric with $\eta_{\hat{\mathrm{m}} \hat{\mathrm{n}}}=\operatorname{diag}(+1,-1 \ldots-1)$ and $\hat{g}=\left|\operatorname{det} \hat{g}_{\hat{\mu} \hat{\nu}}\right|$. The covariant spinor derivative is given by

$$
D_{\hat{\mu}} \psi=\left(\partial_{\hat{\mu}}-\frac{1}{2} \hat{\varepsilon}_{\hat{\mu} \hat{n} \hat{p}} \Sigma^{\tilde{n} \hat{p}}\right)_{\psi}
$$

with the d-dimensional spin connection $\widehat{\omega}_{\hat{\mu}} \hat{n} \hat{p}$ defined by

$$
\begin{aligned}
& \hat{\omega}_{\hat{\mu} \hat{m} \hat{p}}=\hat{e}_{\hat{\mu}} \hat{m} \hat{\omega}_{\hat{m} \hat{n} \hat{p}}
\end{aligned}
$$

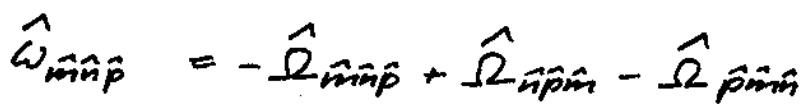

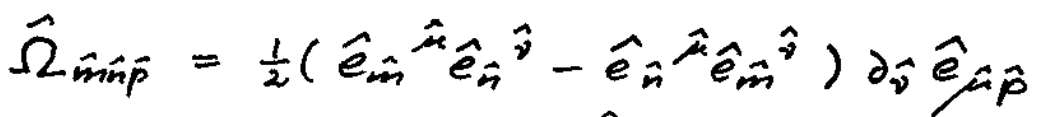

The d-dimensional Dirac matrices $\gamma^{\hat{\mathrm{m}}}$ fulfil the commutation relations

$$
\left\{\gamma^{\hat{m}}, \gamma^{\hat{n}}\right\}=2 \eta^{\hat{m} \hat{n}}, \quad \Sigma^{\hat{m} \hat{n}}=-\frac{1}{4}\left[\gamma^{\hat{m}}, \gamma^{\hat{n}}\right]
$$

The spinor $\bar{\psi}$ is defined by

$$
\begin{array}{ll}
\bar{\psi}=\psi^{+} \gamma^{0} & \text { for Dirac and Weyl spinors } \\
\bar{\psi}=\psi^{T} C_{d} & \text { for Majorana and Majorana-Weyl spinors }
\end{array}
$$

with

$$
\left(\Sigma^{\hat{m} \hat{n}}\right)^{T}=-C_{d} \Sigma^{\hat{m} \hat{m}} C_{d}^{-1}
$$

[For details on Majorana and WeyI spinors in arbitrary dimensions, see ref. 8 . We use the conventions of this paper*).] The purely gravitational part of the

*) We profit from this occasion to correct a typing error in this paper. In eq. (29) $\varepsilon_{1}, \varepsilon_{2}$ should be replaced by $\delta_{1}, \delta_{2}$. 
action is determined by $f$, a scalar function of $\hat{g}_{\hat{\mu} \hat{\nu}}$ and $i t s$ derivatives. Typically, f contains invariants formed from combinations of the d-dimensional curvature tensor $\hat{k}_{\hat{\mathrm{m}} \hat{n} \hat{p} \hat{\mathrm{q}}}$

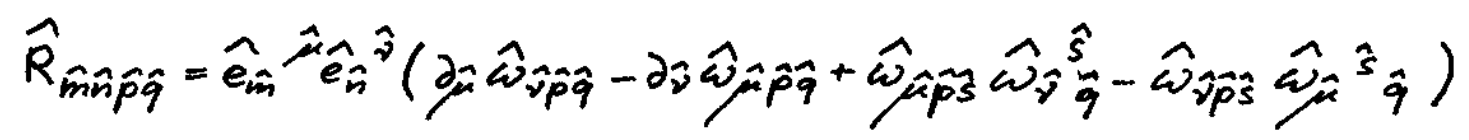

or Ricci tensor

$$
\hat{R}_{\hat{m} \hat{p}}=\hat{R}_{\hat{m} \hat{n} \hat{p} \hat{q}} \eta^{\hat{n} \hat{q}}
$$

like the curvature scalar

$$
\hat{R}=\hat{R} \hat{m} \hat{p} \eta^{\hat{m} \hat{p}}
$$

or higher derivative terms like $\widehat{\mathrm{R}}_{\hat{\mathrm{m}} \mathrm{n}} \widehat{\mathrm{R}}^{\mathrm{m}} \hat{\mathrm{n}} \hat{\mathrm{n}}$, but we also could include covariant derivatives of the curvature tensor or torsion. For the discussion of this and the next three sections we do not consider contractions with the d-dimensional totally antisymetric $\varepsilon$-tensor. (See, however, the remarks in Section 6.)

This action exhibits discrete symmetries of three possible types:

i) discrete symmetries which are contained in the general coordinate transformations;

ii) discrete symetries contained in Spin(d - 1, 1);

iii) discrete symmetries not contained in gen $\mathrm{d}^{\text {. }}$ of course, combinations of the three types of discrete symmetries are also possible.

The first type of symmetries includes reflections in an arbitrary number of coordinates. We denote by $\mathrm{I}_{\hat{v}}$ a reflection of the coordinate $x^{\hat{v}}$ :

$$
I_{\hat{\nu}}: x \rightarrow x^{\prime}
$$

with

$$
\begin{aligned}
& x \hat{\nu} \rightarrow-x^{\hat{\nu}} \\
& x^{\hat{\rho}} \rightarrow x^{\hat{\rho}}, \quad \hat{\rho} \neq \hat{\gamma}
\end{aligned}
$$

Corresponding1y, all covariant and contravariant tensors are multiplied by a factor -1 for every tensor index $\hat{\nu}$ : 


$$
\begin{aligned}
& \hat{g}_{\hat{\nu} \hat{\nu}}(x) \rightarrow \hat{g}_{\nu \hat{\nu}}\left(x^{\prime}\right) \\
& \hat{g}_{\hat{\nu} \hat{\rho}}(x) \rightarrow-\hat{g}_{\nu} \hat{\rho}\left(x^{\prime}\right) \\
& \hat{g}_{\hat{\rho} \hat{\sigma}}(x) \rightarrow \hat{g}_{\hat{\rho} \hat{\sigma}}\left(x^{\prime}\right), \hat{\rho} \neq \hat{v}, \hat{\sigma} \neq \hat{\nu}
\end{aligned}
$$

of course, all $\partial_{\hat{v}}$ derivatives also change sign and the scalars (with respect to coordinate transformations) $\psi, \hat{\omega}_{\hat{\mathrm{m}} \hat{n} \hat{p}}$ and $\hat{\mathrm{R}}_{\hat{\mathrm{m}} \hat{\mathrm{n}} \hat{p} \hat{\mathrm{q}}}$ transform as

$$
\begin{aligned}
& \psi(x) \rightarrow \psi\left(x^{\prime}\right) \\
& \hat{\omega}_{\min \hat{p}}(x) \rightarrow \hat{\omega}_{\min \hat{p}}\left(x^{\prime}\right) \\
& \hat{R}_{\min \hat{p} \hat{q}}(x) \rightarrow \hat{R}_{\min \hat{p} \hat{q}}\left(x^{\prime}\right)
\end{aligned}
$$

Special reflections of this kind are the time reflection

$$
T_{x}=I_{\hat{o}}
$$

and the coordinate parity

$$
P_{x}=I_{\hat{n}} I_{\hat{z}} \ldots I_{d \hat{-1}}
$$

General coordinate invariance guarantees the invariance of the action with respect to these discrete symmetries. However, for any ground state, which is characterized by a fixed value of the vielbein, $\stackrel{\circ}{\hat{\mu}}_{\hat{\mu}}(x)$, the discrete coordinate reflections are broken, since $e_{\hat{\mu}}^{\hat{m}}$ transforms non-trivially with respect to these reflections. Only combinations of discrete coordinate transformations with symmetries of the type (ii) or (iii) may be conserved.

Under infinitesimal d dimensional Lorentz transformations, the spinor and the vielbein transform according to

$$
\begin{aligned}
& \psi(x) \rightarrow \psi^{\prime}(x)=\left(1-\frac{1}{2} \sum_{\min }(x) \Sigma^{\hat{m} \hat{n}}\right) \psi(x) \\
& \hat{e}_{\hat{\mu}}^{\hat{m}}(x) \rightarrow \hat{e}_{\hat{n}}^{\hat{n}}(x)=\left(\delta_{\hat{n}}^{\hat{m}}+\varepsilon^{\hat{m}} \hat{n}(x)\right) \hat{e}_{\hat{n}}^{\hat{n}}(x)
\end{aligned}
$$

with antisymmetric transformation parameters $\varepsilon_{\hat{\mathrm{m}} \hat{\mathrm{n}}}=-\varepsilon_{\hat{\mathrm{n}} \hat{\mathrm{m}}}$. A finite rotation in the m̂ñ plane with rotation parameter $\alpha=-\varepsilon_{\hat{\mathrm{n}} \hat{n}}$ can be written as 


$$
\begin{aligned}
\psi^{\prime}(x) & =\exp \left(\alpha \Sigma^{\hat{m} \hat{n}}\right) \psi(x) \\
& =\left(\cos \frac{\alpha}{2}+\sin \frac{\alpha}{2} \cdot\left(2 \Sigma^{\hat{m} n}\right)\right) \psi(x)
\end{aligned}
$$

if $\hat{\mathrm{m}}$ and $\hat{\mathrm{n}}$ denote both spacelike directions. In this case one has

$$
\begin{aligned}
& \varepsilon \hat{m}^{\hat{n}}=-\varepsilon_{\hat{n}} \hat{m}
\end{aligned}
$$

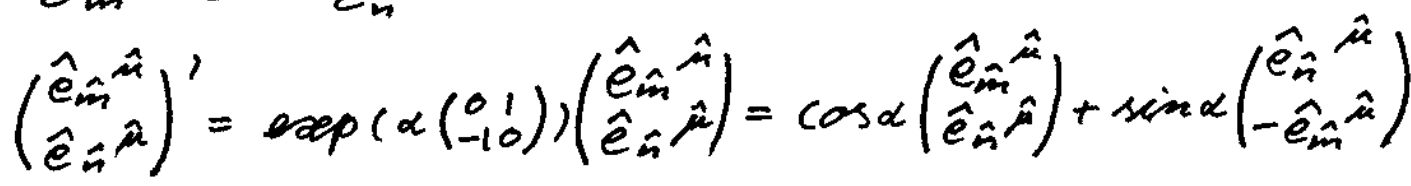

The discrete symmetry $I_{\hat{\mathrm{a}} \hat{\mathrm{n}}}^{\ell}$ is a rotation with parameter $\alpha=-\pi$ :

$$
\begin{aligned}
I_{\dot{m} \hat{n}}^{\mathscr{L}}: & \hat{e}_{\hat{m}}^{\hat{\mu}}(x) \rightarrow-\hat{e}_{\hat{m}}^{\hat{\mu}}(x) \\
& \hat{e}_{\hat{n}} \hat{\mu}(x) \rightarrow-\hat{e}_{\hat{n}} \hat{\mu}(x) \\
& \hat{e}_{\hat{p}}^{\hat{\mu}}(x) \rightarrow \hat{e}_{\hat{p}}(x), \hat{p} \neq \hat{m}, \hat{n} \\
& \rightarrow(x) \rightarrow \gamma^{\hat{m}} \gamma^{\hat{n}} \psi(x)
\end{aligned}
$$

All tensor components with an odd number of indices $\hat{\mathrm{m}}$ or $\hat{\mathrm{n}}$ change sign and the action is invariant under all combinations of transformations If If vielbein characterizing the ground state $\stackrel{0}{\hat{\mu}}_{\hat{\mu}}$ is independent of two coordinates $\mathrm{x}^{\hat{\mu}_{0}}, \mathrm{x}^{\hat{\nu}_{0}}$ and diagonal in these directions

$$
\dot{e}_{\hat{\mu}_{0}}^{\hat{p}} \sim \delta_{\hat{\mu}_{0}}^{\hat{p}}, \dot{e}_{\hat{v}_{0}}^{\hat{p}} \sim \delta_{\hat{\psi}}^{\hat{p}}, \dot{e}_{\mu}^{\hat{m}} \sim \delta_{\hat{\mu}}^{\hat{m}}, \dot{e}_{\hat{\mu}}^{\hat{n}} \sim \delta_{\hat{\mu}}^{\hat{m}}
$$

then the ground state is invariant under the combined reflection $I_{\hat{\nu}} I_{\hat{\mu}} I_{\hat{m} \hat{n}}^{\ell}$. For example, Minkowski space with $\stackrel{o}{\hat{\mu}}_{\hat{\mu}}^{\hat{m}}=\delta_{\hat{\mu}}^{\hat{m}}$ is invariant under the combined reflection of two spacelike coordinates.

However, we note that a reflection of only one Lorentz-index is not contained in the Lorentz rotations, nor is the reflection of one timelike and one spacelike index a Lorentz symmetry. If such symmetries exist, they must belong to symetries of the type (iii). Such symmetries are not contained in gen $\mathrm{d}_{\mathrm{d}}$ and they are therefore not necessarily symetries of the action. As a consequence, reflection in the observed three spacelike coordinates or time reflection are not necessarily symmetries of the reduced four-dimensional action, even if the ground state with respect to the four observed coordinates is Minkowski space and 
therefore invariant with respect to these symmetries. Since pure gravitational theories omitting spinor fields are trivially invariant with respect to a reflection of one Lorentz index $I_{\hat{\mathrm{m}}}^{\ell}$

$$
\begin{aligned}
I_{\hat{m}}^{L}: & \hat{e}_{\dot{m}}^{\hat{\mu}}(x) \rightarrow-\hat{e}_{\dot{m}}^{\hat{\mu}}(x) \\
& \hat{e}_{\hat{p}} \hat{\mu}(x) \rightarrow \hat{e}_{\hat{p}} \hat{\mu}(x), \hat{p} \neq \hat{m}
\end{aligned}
$$

all violations of four-dimensional parity must therefore be due to the couplings of spinor fields.

Let us first consider symetries of the type (iii) for one d-dimensional Dirac spinor. The reflection of one spacelike Lorentz index is then achieved by the transformation

$$
\begin{aligned}
& I_{\hat{m}}^{\mathscr{L}}: \psi \rightarrow \eta_{a} \gamma^{\hat{m}} \bar{\gamma} \psi \quad \text { for } \mathrm{d} \text { even } \\
& \psi \rightarrow \eta_{a} \gamma^{\hat{m}} \psi \text { for dodd }
\end{aligned}
$$

where $\eta_{a}$ is a phase and

$$
\bar{\gamma}=\eta_{\gamma} \gamma^{0} \gamma^{1} \cdots \gamma^{\alpha-1} \quad, \bar{\gamma}^{2}=1
$$

The invariance of the action under the transformation (19), (20) is easily checked. Starting with a d-dimensional Dirac spinor and assuming a ground state $\mathrm{M}^{4} \times \mathrm{K}^{\mathrm{D}}$, the reduced four dimensional theory is invariant under the following reflection of the three spacelike Minkowski coordinates:

$$
P_{3}=I_{\hat{\imath}} I_{\hat{\imath}} I_{\hat{3}} I_{\hat{1}}^{\mathscr{2}} I_{\hat{2}}^{\mathscr{2}} I_{\hat{3}}^{\mathscr{L}}
$$

The product of two reflections $I_{\hat{\mathrm{m}}}^{\ell}$ and $\mathrm{I}_{\hat{\mathrm{n}}}^{\ell}$ applied on spinors is a Lorentz transformation

$$
I_{\hat{n}}^{\mathscr{2}} I_{\dot{n}}^{\mathscr{L}}= \pm I_{\hat{m} \hat{n}}^{\mathscr{2}}
$$

and we have the following commutation relations

$$
\left\{I_{\dot{m}}^{\ell}, I_{\hat{n}}^{\mathscr{L}}\right\}=0,\left\{I_{\hat{m}}^{\mathcal{L}}, I_{\dot{m} \hat{n}}^{\ell}\right\}=0,\left[I_{n}^{\ell}, I_{n \hat{p}}^{l}\right]=0, \hat{m} \neq \hat{n} \neq \hat{p}
$$

[Applied on the vielbein, all $I_{\hat{\mathrm{m}}}^{\ell}$ commute and $I_{\hat{\mathrm{m}}}^{\ell} L_{\hat{\mathrm{n}}}=I_{\hat{\mathrm{m}} \hat{\mathrm{n}}}^{\ell}$, corresponding to the two to one covering of $\mathrm{SO}(\mathrm{d}-1,1)$ by the group $\operatorname{Spin}(\mathrm{d}-1,1)$.$] The d-dimensional$ parity operation $P_{d}$ is defined by 


$$
P_{d}=P_{x} P_{\mathscr{L}}
$$

with

$$
P_{\mathscr{R}} \psi=\eta_{P} \gamma^{0} \psi
$$

and

$$
\prod_{\hat{m} \neq 0} I_{\hat{m}}^{\mathscr{L}}= \pm P_{\mathscr{L}}
$$

For d odd, $\mathrm{P}_{\mathcal{L}}$ is a pure Lorentz transformation.

Similarily, we can define for Dirac spinors a d-dimensional time reversal operation:

$$
T_{d}=T_{x} T_{\mathscr{E}}
$$

with

$$
\begin{aligned}
& T_{\mathscr{L}} \hat{e}_{0}^{\hat{\mu}}=-\hat{e}_{0}^{\hat{\mu}}, T_{\mathscr{L}} \hat{e}_{\dot{m}}^{\hat{\mu}}=\hat{e}_{m}^{\hat{\mu}}, \hat{m} \neq 0 \\
& T_{\mathscr{L}} \psi=\eta_{T} \gamma^{0} \bar{\gamma} B_{d}^{-1} \psi * \quad \text { for d even } \\
& T_{\mathscr{L}} \psi=\eta_{T} \gamma^{0} B_{d}^{-1} \psi^{*} \quad \text { for d odd }
\end{aligned}
$$

Here $n_{T}$ is again a phase and $B_{d}$ is given by $B_{1 d}$ for $d=2,3,4,7 \bmod 8$ and by $\mathrm{B}_{2 \mathrm{~d}}$ for $\mathrm{d}=5,6,8,9 \bmod 8$ obeying

$$
\begin{aligned}
& \left(\gamma^{\hat{m}}\right)^{*}=-B_{1 d} \gamma^{\hat{m}} B_{1 d}{ }^{-1} \\
& \left(\gamma^{\hat{m}}\right)^{*}=B_{2 d} \gamma^{\hat{m}} B_{2 d d^{-1}}
\end{aligned}
$$

$\mathrm{T}_{\boldsymbol{\ell}}$ involves a complex conjugation and acts as an antilinear operator. Furthermore, $\mathrm{T}_{\boldsymbol{\ell}}$ involves a total reordering of all fields which is equivalent to a transposition:

$$
T_{\mathscr{L}}\left(\psi_{\alpha} \psi_{\alpha}\right)=\left(T_{\mathscr{L}} \psi_{/ \beta}\right)\left(T_{\mathscr{L}} \psi_{\alpha}\right)=-\left(T_{\mathscr{L}} \psi_{\alpha}\right)\left(T_{\mathscr{L}} \psi_{/}\right)
$$

Symetries of the type (iii) contain also discrete symmetries leaving the vielbein and therefore the gravitational part of the action invariant. One such symetry is d-dimensional charge conjugation $e_{d}$ :

$$
\begin{aligned}
& \varphi_{d} \psi=B_{d}^{-1} \psi^{*}=\psi^{c} \\
& \mathscr{C}_{d} \hat{e}_{\hat{m}} \hat{\mu}=\hat{e}_{\hat{m}} \hat{m}
\end{aligned}
$$


$-11-$

The charge conjugate spinor $\psi^{c}$ has the same gen ${ }_{d}$ transformation properties (15) as $\psi$,

$$
\left[\mathscr{C}_{d}, \Sigma^{\hat{m} \hat{n}}\right]=\left(B_{d}^{-1}\left(\Sigma^{\hat{m} \hat{n}}\right)^{*}-\Sigma^{m n} B_{d}^{-1}\right)(*)=0
$$

where the symbol (*) denotes a complex conjugation of all fields on which $e_{d}$ operates. The invariance of the action (1) under $\mathcal{C}_{d}$ is easily verified.

Finally, for Dirac spinors and $d$ even, we define the discrete symmetry $\Gamma$ :

$$
\Gamma: \psi \rightarrow \overline{\gamma \psi}, \hat{e}_{m}^{\hat{\mu}} \rightarrow \hat{e}_{m} \hat{\mu}
$$

Again, $\Gamma$ commutes with gen transformation

$\left[\Sigma^{\hat{n} \hat{n}}, \bar{\gamma}\right]=0$

and it is a symmetry of the action (1). It distinguishes the different Wey1 spinous

$$
\begin{array}{ll}
\psi_{+}=\frac{1}{2}(1+\bar{\gamma}) \psi & , \bar{\gamma} \psi_{+}=\psi_{+} \\
\psi=\frac{1}{2}(1-\bar{\gamma}) \psi & , \bar{\gamma} \psi_{-}=-\psi_{-}
\end{array}
$$

For $d=4 \bmod 4, e_{d}$ anticommutes with $\Gamma$ and for $d=2 \bmod 4 \mathfrak{e}_{d}$ and $\Gamma$ commute.

$$
\begin{aligned}
& {\left[\mathscr{C}_{\alpha}, \bar{\gamma}\right]=0 \quad \text { for } \mathrm{d}=2 \bmod 4} \\
& \left\{\mathcal{E}_{\alpha}, \bar{\gamma}\right\}=0 \quad \text { for } \mathrm{d}=4 \bmod 4
\end{aligned}
$$

This corresponds to the wel1-known fact that the inequivalent Wey1 spinous belong to complex representations, conjugate to each other, for $d=4 \bmod 4$, whereas for $d=2 \bmod 4$ Weyl spinors axe real or pseudoreal representations of the Lorentz group.

We finally will choose definite phases $\eta_{a}$, $\eta_{p}$, $\eta_{T}$. First, we require for all discrete transformations $I$ discussed so far, that applying $I$ twice on a spinor field results in an identity transformation up to a minus sign $\left(I^{2}= \pm 1\right)$. This implies $\eta_{\mathrm{a}}^{4}=\eta_{\mathrm{P}}^{4}=\eta_{\mathrm{T}}^{4}=1$, consistent with the requirements (23) and (26). Second, we choose the phases so that the discrete transformations $I_{\hat{\mathrm{m}}}^{\mathcal{L}}, \mathrm{P}_{\boldsymbol{\ell}}$ and $\mathrm{T}_{\boldsymbol{\ell}}$ commute with charge conjugation:

$$
\left[\mathscr{C}_{d}, I_{m}^{k}\right]=0,\left[\varepsilon_{d}, P_{e}\right]=0,\left[\varepsilon_{d}, T_{d e}\right]=0
$$


This will allow us to use the same definition of these transformations for Dirac and for Majorana spinors. The phases are now fixed up to a minus sign and we list the operation on spinors of the discrete transformations, including the combined symetry $(\mathrm{PCT})_{\mathrm{d}}$, in table 1 .

For Dirac spinors, the action (1) has a global U(1) symmetry

$$
\psi \rightarrow \exp (i \phi) \psi
$$

For $d$ even, the global symmetry is $U(1) \times U(1)$ and includes chiral phase rotations

$$
\psi \rightarrow \operatorname{egp}(i \alpha \bar{\gamma}) \psi
$$

A combination of a discrete symmetry with a phase rotation yields of course an equivalent discrete symmetry.

Except for $\mathrm{d}=5,7 \mathrm{mod} 8$, Dirac spinors are not irreducible representations of Spin(d-1,1). The irreducible representations are Majorana-spinors for $\mathrm{d}=3,9 \bmod 8$, Weyl spinors for $\mathrm{d}=4,6,8 \bmod 8$ (for $\mathrm{d}=4 \bmod 4$, Majorana and Weyl spinors are equivalent) and Majorana-Weyl spinors for $d=2$ mod 8 . For Majorana and Weyl spinors the symmetries $\mathcal{C}_{d}$ and $\Gamma$ reflect the existence of more than one irreducible representation in the action. Majorana-spinors are eigenstates of charge conjugation

$$
\varepsilon_{d} \psi_{M}=B_{d}{ }^{-1} \psi_{M}^{*}=\psi_{M}
$$

Since $\mathcal{C}_{\mathrm{d}}$ commutes with $\mathrm{I}_{\hat{\mathrm{m}}}^{\ell}, \mathrm{P}_{\mathcal{\ell}}$ and $\mathrm{T}_{\mathcal{\ell}}$, these transformations are still defined for Majorana spinors. The action is invariant under these symmetries, noting that for Majorana spinors one has the identity

$$
\bar{\psi}_{\mu}=\psi_{\mu}{ }^{+} \gamma^{0}=\psi_{\mu}{ }^{T} B_{d} \gamma^{0}=\psi^{T} C_{d}
$$

There is no non-trivial d-dimensional charge conjugation for Majorana spinors. Wey1 spinors are eigenstates of $\bar{\gamma}$ :

$$
\bar{\gamma} \psi_{+}=\psi_{+}
$$

The symmetry $\Gamma$ is now trivial. Reflections of an odd number of Lorentz indices anticommute with $\bar{\gamma}$ 


$$
\left\{I_{\text {is }}^{\mathscr{L}}, \bar{\gamma}\right\}=0, \quad\left\{P_{3}, \bar{\gamma}\right\}=0, \quad\left\{P_{\alpha}, \bar{\gamma}\right\}=0
$$

and these operations cannot be defined for Weyl spinors. As we11, due to the commutation relations (36), the charge conjugation operation cannot be defined for Weyl spinors in $d=4 \bmod 4$ whereas for Weyl spinors in $d=2 \bmod 4, e_{d}$ is still a symmetry of the action. For $d=4 \bmod 4$, the combined symmetries $\mathfrak{C}_{d} I_{\hat{m}}$, $e_{d} P_{3}, e_{d} P_{d}$ commute with $\bar{\gamma}$ and are symmetries of the action. These symmetries are not defined for Weyl spinors in $d=2 \bmod 4$. The situation is similar for time reversal. One has

$$
\begin{aligned}
& {\left[\bar{\gamma}, T_{d}\right]=0 \quad \text { for } \mathrm{d}=4 \bmod 4} \\
& \left\{\bar{\gamma}, T_{d}\right\}=0 \text { for } \mathrm{d}=2 \bmod 4
\end{aligned}
$$

and $\mathrm{T}_{\mathrm{d}}$ is a symmetry for Weyl spinors only for $\mathrm{d}=4 \bmod 4$. Finally, MajoranaWeyl spinors are the irreducible spinor representations for $\mathrm{d}=2$ mod 8 . They are eigenstates of both $\bar{\gamma}$ and $e_{d}$ :

$$
\varphi_{d} \psi_{M+} \equiv \bar{\gamma} \psi_{M+} \equiv \psi_{M+}
$$

The only non-trivial symetry of type (iii) for Majorana-Weyl spinors is a combination of time reversal with a reflection of an odd number of Lorentz indices. The action is invariant under symmetries like $I_{\hat{m}^{T}}{ }_{d}, P_{3} T_{d}, P_{d} T_{d}$. In table 2 we list the discrete symmetries for the different irreducible spinor representations of Spin(d - 1, 1). Of course, combinations of these symmetries are also symetries of the action. Let us also remind at this place that the kinetic term for Weyl spinors has a global U(1) invariance of phase transformations. [For the equivalent Majorana spinors in $\mathrm{d}=4 \bmod 4$, this corresponds to transformations $\left.\psi_{\mathrm{M}} \rightarrow \exp (i \alpha \bar{\gamma}) \psi_{\mathrm{M}^{*}}\right]$ No globa1 $\mathrm{U}(1)$ symmetry exists for Majorana-spinors in $\mathrm{d}=3,9 \bmod 8$ or a Majorana-Weyl spinor in $\mathrm{d}=2 \bmod 8$.

3. DIMENSIONAL REDUCTION OF d-DIMENSIONAL DISCRETE SYMMETRIES: REFLECTIONS OF MINKOWSKI COORDINATES AND d-DIMENSIONAL CHARGE CONJUGATION

For a ground state $\mathrm{M}^{4} \times \mathrm{K}^{\mathrm{D}}$, the d-dimensional spinor (as well as the vielbein) decomposes into infinitely many four-dimensional spinors $\phi^{i}(x)$ : 


$$
\psi(y, x)=\sum_{i} \psi_{i}(y) \varphi_{i}(x)
$$

(We denote the coordinates of $\mathrm{K}^{\mathrm{D}}$ by $\mathrm{y}^{\alpha}$ or $\phi^{\alpha}$ and the coordinates of Minkowski space by $x^{\mu}$. For details of the decomposition and notations see ref. 3.) The reduced four-dimensional theory is a gauge theory of spinors coupled to scalars, spin one and spin two bosons. Therefore, the four-dimensional parity $\bar{P}$, time reversal $\bar{T}$ and charge conjugation $\overline{\mathrm{C}}$ involve also non-trivial transformations of the scalar and vector fields. (Transformation properties of spinor bilinears can be found in Section 5.) We note that $\bar{P}, \bar{T}$, and $\bar{C}$ act only on Minkowski coordinates and $\phi_{i}(\mathrm{x})$, but not on $\psi_{i}(\mathrm{y})$ :

$$
\begin{array}{ll}
\bar{P}: \quad & x^{\mu}=\left(x^{*}, x^{i}\right) \rightarrow\left(x^{\mu}\right)^{\prime}=\left(x^{0},-x^{i}\right) \\
& \bar{P} \varphi_{i}(x)=i \gamma^{0} \varphi_{i}\left(x^{\prime}\right) \\
\bar{T}: \quad & x^{\mu} \rightarrow-\left(x^{\mu}\right)^{\prime} \\
& \bar{T} \varphi_{i}(x)=\gamma^{0} \bar{\gamma}^{5} B_{\psi}^{-1} \varphi_{i}^{*}\left(-x^{\prime}\right)=-\gamma^{A} \gamma^{3} \varphi_{i}^{*}\left(-x^{\prime}\right) \\
\bar{C}: \quad x^{\mu} \rightarrow x^{\mu} \\
\bar{C} \varphi_{i}(x)=B_{\psi}^{-1} \varphi_{i}^{*}(x)=i \gamma^{2} \varphi_{i}^{*}(x) \\
\bar{\gamma}=\bar{\gamma}^{5} \overline{\Gamma_{D}} \\
\overline{\gamma^{5}}=i \gamma^{0} \gamma^{1} \gamma^{2} \gamma^{3} \\
\overline{\Gamma_{D}}=\eta_{D} \gamma^{4} \gamma^{5} \ldots \gamma^{\alpha-1}=\eta_{D} \Gamma^{1} \Gamma^{2} \ldots \Gamma^{D}
\end{array}
$$

with $D$ anticommuting Dirac matrices $\Gamma^{a}$ acting on $\psi^{\hat{i}}(y)$, one finds that the $d-$ dimensional Weyl constraint implies a Weyl constraint on the spinors $\phi_{i}$ : Lefthanded spinors $\phi_{i L}$ are obtained from the expansion of $\psi^{+}(y)=1 / 2\left(1+\bar{\Gamma}_{D}\right) \psi(y)$, whereas right-handed spinors $\phi_{i R}$ derive from $\psi^{-}(y)=1 / 2\left(1-\bar{\Gamma}_{\mathfrak{D}}\right) \psi(y)$. The Wey1 spinor $\psi_{+}(\mathrm{y}, \mathrm{x}) \equiv \bar{\gamma} \psi_{+}(\mathrm{y}, \mathrm{x})$ contains both left-handed and right-handed four-dimensional spinors. For Weyl spinors $\phi_{i L}$ the transformations $\bar{P}$ and $\bar{C}$ axe not defined separately; only $\overline{\mathrm{T}}, \overline{\mathrm{CP}}$ and $\overline{\mathrm{CPT}}$ are consistent with the Weyl constraint. However, a left-handed and a right-handed Weyl spinor belonging to the same representation of the gauge group may be combined into a Dirac spinor. (This is the case for a11 massive spinors belonging to complex representations.) Weyl spinors belonging 
to rea1 or pseudoreal representations are equivalent to Majorana spinors, for which $\overline{\mathrm{P}}$ is defined (and equivalent to $\overline{\mathrm{CP}}$ ). If there are left-handed Weyl spinors $\phi_{C L}$ belonging to a complex representation $C$ together with other left-handed spinors $\phi_{\overline{\mathrm{C}} \mathrm{L}}$ belonging to the complex conjugate representation $\overline{\mathrm{C}}$, a non-trivial charge conjugation $\overline{\mathrm{C}}$ can be defined as an exchange of these spinors. (It may be used to combine them into a Dirac spinor.) Furthermore, it may be possible to combine a reflection of the three spacelike coordinates with an outer automorphism of the gauge symmetry. [A typical example is left-right-symmetry in $\mathrm{s} 0(10)$ or $\left.\mathrm{SU}(2)_{\mathrm{L}} \times \mathrm{SU}(2)_{\mathrm{R}} \times \mathrm{U}(1) \times \mathrm{SU}(3)_{\mathrm{C}} \cdot\right]$

Since all these complexions can occur in dimensional reduction, we simplify our discussion by defining classes of discrete transformations for the effective four-dimensional theory. The class $\tilde{\mathrm{P}}$ contains all symmetries containing a reflection of the three spacelike coordinates plus all transformations which can be obtained from such a symmetry by a four-dimensional global Lorentz-rotation or translation. Similarily, the symetries of the class $\tilde{T}$ contain a reversal of time. Then all symmetries belong to one of the four classes $\tilde{\mathrm{I}}$ (no coordinate reflection), $\tilde{\mathrm{P}}, \tilde{\mathrm{T}}$, and $\tilde{\mathrm{PT}}$ (reflection of all Minkowski coordinates). Some elements of these symmetry classes are given in table 3 . We note that there exists a group structure with respect to a multiplication of these classes, with $\tilde{I}$ the identity element and $\tilde{\mathrm{P}}^{2}=\tilde{\mathrm{T}}^{2}=(\tilde{\mathrm{P} T})^{2}=\tilde{\mathrm{I}}$.

After these preparations we now address the question of how the d-dimensional discrete symetries are related to four-dimensional transformations. First we note that the definition of the classes $\tilde{\mathrm{I}}, \tilde{\mathrm{P}}, \tilde{\mathrm{T}}$, and $\tilde{\mathrm{PT}}$ does not refer to dimensional reduction. A symmetry reversing the first three spacelike coordinates in d dimensions does the same for the reduced four-dimensional theory. The ddimensional symmetries $P_{3}, T_{d}, e_{d}, \Gamma$ transform the internal components of the vielbein and the metric trivially. They cannot be broken by spontaneous compactification. [The vacuum value $\stackrel{\circ}{e}_{\alpha}^{a}(y)$ is invariant.] If the symetry $\mathrm{P}_{3}$ can be defined in d-dimensions, the reduced four-dimensional theory necessarily has a conserved symetry of the class $\tilde{P}$. If our model of a spinor and pure gravity 
admits the symmetry $T_{d}$, some symmetry of the class $\tilde{T}$ leaves the four-dimensional action invariant.

This gives severe constraints on acceptable d-dimensional models of pure gravity coupled to spinors. Experimentally, we know that the weak interactions neither conserve $\bar{P}$ nor $\overline{C P}$. The only conserved discrete symmetry for spinors

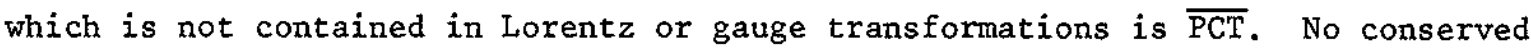
symmetry of the class $\tilde{\mathrm{P}}$ or $\tilde{\mathrm{T}}$ is consistent with experiment. There is no symmetry of the class $\tilde{\mathrm{I}}$ transforming the known spinors non-trivia1ly. (This would imply a non-trivial charge conjugation or a degeneracy of the fermionic mass spectrum.) Reading table 2 one finds: gravity theories in $d=3,4,5,7,8$, 9 reduce to four-dimensional theories with discrete symmetries of the type $\tilde{\mathrm{P}}$ and $\tilde{\mathrm{T}}$. For $\mathrm{d}=5,6,7$ the transformation $C_{d}$ is a symmetry of the class $\tilde{I}$ transforming all four-dimensional spinors non-trivially. The only acceptable possibility in this context is a Majorana-Weyl spinor for $d=2 \bmod 8$. It is interesting to remember that $d=2 \bmod 4$ is the only case where the existence of chiral four-dimensional fermions cannot be excluded by using merely the properties of the clifford algebra $^{3)}$. A Majorana-Weyl spinor in $\mathrm{d}=2 \bmod 8$ induces a non-trivial fourdimensional symmetry of the class $\tilde{P T}$. We will show that this corresponds to the transformation $\overline{\mathrm{PCT}}$.

Let us now look at the symmetries $\mathcal{C}_{d}, P_{3}$ and $T_{d}$ in detail. The transformation $e_{d}$ induces a four-dimensional transformation of the class $\tilde{I}$ :

$$
\begin{aligned}
& \varphi_{\alpha} \psi_{i}(y) \varphi_{i}(x)=B_{D}^{-1} \psi_{i}(y)^{*} B_{\psi}^{-1} \varphi_{i}(x)^{*}=\psi_{i}(y) B_{i j} B_{4}^{-1} \varphi_{g}(x)^{*} \\
& \mathscr{C}_{\alpha} \varphi_{i}(x)=B_{i j} B_{4}^{-1} \varphi_{j}(x)^{*}
\end{aligned}
$$

(For $d=5,6,8,9 \bmod 8, B_{4}^{-1}$ has to be replaced by $\bar{\gamma}^{5} \mathrm{~B}_{4}^{-1}$.) If $\psi_{i}(y)$ corresponds to a D-dimensional Majorana spinor, one has $B_{i j}=\delta_{i j}$ and $e_{d}$ corresponds either to $\overline{\mathrm{C}}$ or to $\bar{\gamma}^{5} \overline{\mathrm{C}}$. Otherwise, $\mathrm{B}_{i j}$ involves an exchange of different four-dimensional spinors $\phi_{i}$ and $\phi_{j}$ with equal mass and belonging to representations of the gauge group which are complex conjugate to each other. $e_{d}$ transforms $\phi_{i}$ into the charge conjugate of $\phi_{j}$ which belongs to the same representation as $\phi_{i}$. Therefore $e_{d}$ has 
not the properties of usual four-dimensional charge conjugation which changes the sign of all abelian charges and transforms every representation into its complex conjugate. $e_{d}$ can be interpreted as a four-dimensional charge conjugation only for real and pseudoreal representations. For complex representations $e_{d}$ exchanges two different spinors with degenerate mass. Clearly, such a symetry does not exist in nature and we must restrict acceptable models to d-dimensional Majorana spinors for which $e_{d}$ is trivial. In this case we have four-dimensional Majorana spinors for the case $B_{i j}=\delta_{i j}$. Otherwise, the spinor $B_{i j} B_{4}^{-1} \phi_{j}(x)$ is identified with $\phi_{i}(x)$ and $\phi_{j}(x)$ is not an independent spinor anymore.

A reflection of the first three spacelike coordinates reads in four dimensions:

$$
\begin{aligned}
& P_{3} \psi_{i}(y) \varphi_{i}(x)=\eta_{3} \bar{\Gamma}_{D} \psi_{i}(y) \gamma^{0} \varphi_{i}\left(x^{\prime}\right)=\eta_{3} \psi_{i}(y) \Gamma_{i j} \gamma^{0} \varphi_{f}\left(x^{\prime}\right) \\
& P_{3} \varphi_{i}(x)=\eta_{3} \Gamma_{i g} \gamma^{0} \varphi_{j}\left(x^{\prime}\right) \quad \text { for d even } \\
& P_{3} \psi_{i}(y) \varphi_{i}(x)=-\eta_{3} \psi_{i}(y) \bar{\gamma}^{5} \gamma^{0} \varphi_{i}\left(x^{\prime}\right) \\
& P_{3} \varphi_{i}(x)=-\eta_{3} \bar{\gamma}^{5} \gamma^{0} \varphi_{i}\left(x^{\prime}\right) \quad \text { for d odd }
\end{aligned}
$$

The phase $\eta_{3}$ can be found in table 1 . For massless four-dimensional spinors $\Gamma_{i j}$ can be chosen diagonal: $\Gamma_{i j}=\delta_{i j}$. For massive spinors $\Gamma_{i j}$ transforms between two different spinors with equal mass (up to an irrelevant minus sign). Clearly, a symmetry of the type $P_{3}$ is not observed in nature and we have to start with a Weyl spinor in $d$ dimensions for which $P_{3}$ is not defined. Since for Weyl spinors in $d=4 \bmod 4$ the symmetry $e_{d} P_{3}$ is defined, we again end with an unacceptable symetry of the class $\tilde{\mathrm{P}}$ in four dimensions. Only Weyl spinors in $\mathrm{d}=2 \bmod 4$ are left as possible candidates for theories of pure gravity without some kind of parity conservation in four dimensions.

Time reversal in dimensions reduces to a symetry of class $\tilde{\mathrm{T}}$ in four dimensions: 
$T_{d} \psi_{i}(y) \varphi_{i}(x)=\eta_{T} \bar{\Gamma} B_{D}^{-1} \psi_{i}(y)^{*} \gamma^{0} \bar{\gamma}^{5} B_{L}^{-1} \varphi_{i}^{*}\left(-x^{\prime}\right)=\eta_{T} \psi_{i}(y) B_{i j} \Gamma_{j} \bar{T} \varphi_{z}(x)$ $T_{d} \varphi_{i}(x)=\eta_{T} B_{i f} \Gamma_{f \&} \bar{T} \varphi_{\xi}(x)$ for d even

$\bar{\zeta}_{d} \psi_{i}(y) \varphi_{i}(x)=\eta_{T} B_{i}^{-1} \psi_{i}(y)^{*} \gamma \bar{\gamma}^{5} B_{4}^{-1} \varphi_{i}^{*}\left(-x^{\prime}\right)=\eta_{T} \psi_{i}(g) B_{i j} \bar{T} \varphi_{g}(x)$

$T_{d} \varphi_{i}(x)=\eta_{T} B_{i g} \bar{T} \varphi_{g}(x) \quad$ for d odd

For $d=3,6,7,8$ mod 8 one should replace in eq. (53) $B_{4}^{-1}$ by $\bar{\gamma}^{5} B_{4}^{-1}$ and $\overline{\mathrm{T}}$ by $\bar{\gamma}^{5} \overline{\mathrm{T}}$.

We note that $\mathrm{T}_{d}$ can correspond to a usual four-dimensional time reversal only for real or pseudoreal representations when $B_{i j}$ is diagonal. For complex representations $B_{i j}$ transforms a spinor into an other spinor belonging to the complex conjugate representation. $T_{d}$ behaves rather like a four-dimensional $\overline{C T}$ transformation.

Indeed, if a gauge group admits complex representations [this is the case of electromagnetism or $\mathrm{SU}(3)]$, we can find another classification of discrete symmetries in four dimensions: symmetries which transform every field belonging to a complex representation into a field belonging to the complex conjugate representation are in the class $\stackrel{\circ}{\mathrm{C}}$. This class includes four-dimensional charge conjugation, $\overline{\mathrm{CP}}$ and $\overline{\mathrm{PCT}}$. Symmetries which do not change the representation with respect to the gauge group belong to the class $\stackrel{\circ}{\mathrm{I}}$. This classification can be combined with the classes $\tilde{\mathrm{I}}, \tilde{\mathrm{P}}, \tilde{\mathrm{T}}$, and $\tilde{\mathrm{PT}}$. For example, $\overline{\mathrm{CP}}$ transformations belong to the classes $\tilde{P}$ and $\&$. The symmetry $T_{d}$ is in the class $\tilde{T} \mathcal{C}$, whereas $P_{3}$ and $e_{d}$ belong to $\tilde{\mathrm{P}} \stackrel{\circ}{\mathrm{I}}$ and $\tilde{\mathrm{I}} \mathrm{I}$.

The transformation $\mathrm{P}_{3} \mathrm{~T}_{\mathrm{d}}$ belongs to the class $\tilde{\mathrm{PTC}}$ which is the class of $\overline{\mathrm{PCT}}$ transformations. We note that this transformation is a symetry of the action for all Spin $(d-1,1)$ representations in arbitrary dimensions. (For Weyl spinors in $d=4 \bmod 4 P_{3}$ should be replaced by $e_{d} P_{3}$.) One has

$$
\begin{aligned}
P_{3} T_{d} \psi_{i}(y) \varphi_{i}(x) & =\eta_{3} \eta_{T} B_{D}^{-1} \psi_{i}(y)^{*} \bar{\gamma}^{5} B_{4}^{-1} \varphi_{i}^{*}(-x) \\
& =-i \eta_{3} \eta_{T} \psi_{i}(y) B_{i j} \bar{P} \bar{T} \varphi_{j}(x)
\end{aligned}
$$

$$
P_{3} T_{d} \varphi_{i}(x)=-i \eta_{3} \eta_{T} B_{i j} \bar{P} \bar{T} \varphi_{j}(x)
$$


where $B_{4}^{-1}$ and $\bar{T}$ should be replaced by $\bar{\gamma}^{5} B_{4}^{-1}$ and $\bar{\gamma}^{5} \bar{T}$ for $d=5,6,8,9 \bmod 8$. For d-dimensional Majorana spinors one has $B_{i j} \phi_{j} \equiv B_{4}^{-1} \phi_{i}=\bar{C} \phi_{i}$ (with $\bar{\gamma}^{5}$ multiplication for $d=5,6,8,9 \bmod 8$ ) and therefore

$$
P_{3} T_{d} \varphi_{i}(x)=-i \eta_{3} \eta_{T} \vec{P} \bar{C} \vec{T} \varphi_{i}(x)
$$

which identifies $P_{3} T_{d}$ with the only allowed non-trivial discrete symmetry for four-dimensional spinors.

\section{REFLECTIONS OF INTERNAL COORDINATES}

In this section we will discuss reflections of coordinates of the internal space. Consider a reflection in one of the internal coordinates which we will denote by $\phi$ :

$$
\begin{aligned}
I_{\varphi}: \quad & \phi \rightarrow-\phi \\
& y^{\alpha} \rightarrow y^{\alpha}, \quad y^{\alpha} \neq \phi
\end{aligned}
$$

$I_{\phi}$ is always a symmetry of the action. Suppose for a moment that we can find a corresponding reflection of Lorentz indices $x_{1}^{\ell}$ and that the ground state is invariant with respect to the combined transformation

$$
I_{1}=I_{1}^{\mathscr{L}} I_{\varphi}
$$

Then $I_{1}$ will result in a non-trivial transformation of the class $\tilde{I}$ in four dimensions. Furthermore, the transformation $P_{3} I_{1}$ is always a symmetry of the action

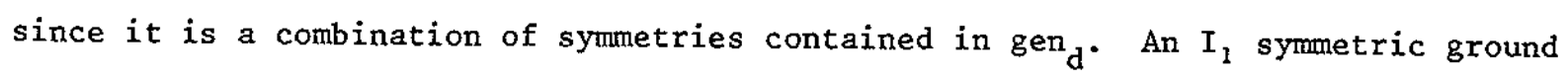
state induces a four-dimensional theory with a $\tilde{\mathrm{P}}$ symmetry. Thus even theories with Weyl spinors in 2 mod 4 dimensions, where neither $I_{1}^{\ell}$ nor $e_{d} I_{1}^{\ell}$ can be defined, lead to unacceptable four-dimensional theories if the ground state is symmetric under $I_{1}$. (Remember that the action of $I_{1}$ on the vielbein can always be defined.) Similar arguments hold for the transformation $I_{1} T_{d}$ or $I_{1} e_{d} T_{d}$. The reduced theory corresponding to an $I_{1}$ symmetric ground state has a conserved symmetry of the class $\tilde{\mathrm{T}}$.

However, there is an important difference between symmetries of the type $I_{1}$ and symmetries discussed in Section 3: symmetries like $P_{3}, T_{d}$ and $C_{d}$ are never broken by a ground state of the form $M^{4} \times k^{D}$. On the contrary, reflections of 
internal coordinates may be symmetries of the action, but not symmetries of the ground state $\mathrm{K}^{\mathrm{D}}$. They can be broken by spontaneous compactification: And we wil1 show that only internal spaces which break all possible non-trivial internal coordinate reflections can lead to realistic models without unobserved non-trivial symmetries of the classes $\tilde{\mathrm{P}}, \tilde{\mathrm{T}}$, or $\tilde{\mathrm{I}}$ in four dimensions!

The action of internal coordinate reflections is closely related to the gauge group of $\mathrm{K}^{\mathrm{D}}$, since the gauge group is a subgroup of general coordinate and Lorentz transformations in $\mathrm{D}$ dimensions $\left(\right.$ gen $\left._{D}\right)$. Before proceeding to a more detailed description of symmetries of the type $I_{1}$, we briefly have to review how gauge transformations act on the vielbein and the spinor. An infinitesimal gauge transformation reads (with $a, b$ "internal" Lorentz indices)

$$
\begin{gathered}
\delta_{G} \hat{e}_{\alpha}^{a}=-\theta^{z}\left(S_{z}\right)_{\alpha b}^{\beta a} \hat{e}_{\beta}^{b} \\
\left(S_{z}\right)_{\alpha b}^{\beta a}=\delta_{b}^{a} \partial_{\alpha} k_{z}^{\beta}+\delta_{b}^{a} \delta_{\alpha}^{\beta} K_{z}^{\gamma} \partial_{\gamma}+\delta_{\alpha}^{\beta} \varepsilon_{z}^{a} b \\
\delta_{G \psi} \psi-\theta^{2} S_{z} \psi \\
S_{z}=K_{z}^{\alpha} \partial_{\alpha}-\frac{1}{2} \varepsilon_{z a b} \sum^{a b}
\end{gathered}
$$

$\theta_{z}(x)$ are the infinitesimal transformation parameters (their number is equal to the dimension of the gauge group $G)$ and $\mathrm{K}_{z}^{\alpha}(\mathrm{y})$ are the corresponding Killing vectors. The antisymmetric functions $\stackrel{\circ}{\varepsilon}_{z a b}(y) \equiv-\stackrel{\circ}{\varepsilon}_{z b a}$ are given by

$$
\dot{\varepsilon}_{z a b}(y)=\dot{e}_{a}^{\alpha} \dot{e}_{\beta b} \partial_{\alpha} K_{z}^{\beta}+\dot{e}_{a}^{\alpha} K_{z} \beta \partial_{\beta} \dot{e}_{\alpha b}
$$

and by definition of the Killing vectors the vielbein $\stackrel{\circ}{e}_{\alpha}^{a}(y)$ and the metric $\stackrel{\circ}{g}_{\alpha \beta}(y)$ of the ground state $\mathrm{K}^{\mathrm{D}}$ are left invariant by the transformations (58). If the D-dimensional spinors $\psi_{\mathrm{nH}}(y)$ are the components of a representation $\mathrm{H}$ of $\mathrm{G}$, they transform according to

$$
S_{z} \psi_{n H}(y)=\psi_{n^{\prime} H}(y)\left(T_{z}\right)_{n^{\prime} n}
$$

with constant matrices $\mathrm{T}_{z} \cdot$ Both $\mathrm{S}_{z}$ and $\mathrm{T}_{z}$ fulfil the commutation rules of the group

$$
\begin{aligned}
& {\left[S_{x}, S_{y}\right]=\rho_{x y z} S_{z}} \\
& {\left[T_{x}, T_{y}\right]=\rho_{x y z} T_{z}}
\end{aligned}
$$


The generators $T_{z}$ describe the action of the gauge group on the four-dimensional spinors $\phi_{\mathrm{nH}}(\mathrm{x})$

$$
\begin{aligned}
& \delta_{G} \psi(y, x)=\delta_{G} \psi_{n H \varepsilon}(y) \varphi_{n H \varepsilon}(x) \\
& =-\theta^{z} S_{z} \psi_{n H \varepsilon}(z) \varphi_{n H \varepsilon}(x)=\psi_{n H \xi}(y)\left\{-\theta^{z}\left(T_{z}\right)_{n n^{\prime}} \Phi_{n^{\prime} H \varepsilon}(x)\right\}
\end{aligned}
$$

[Sumation over all representations $\mathrm{H}$ and all components $\mathrm{n}$ is implied. The index $k$ counts how many times a representation $H$ is contained in $\psi(y, x)$.

Suppose that $\mathrm{K}^{\mathrm{D}}$ admits a continuous symmetry group $\mathrm{G}$ and consider an arbitrary $U(1)$ subgroup of $G$. We parametrize the orbit of this $U(1)$ subgroup by a coordinate $\phi$. [This is always possible except for the points where $U(1)$ acts trivially.] Since this orbit is closed, $\phi$ is periodic $-\pi \leq \phi<\pi$ with $-\pi$ and $\pi$ identified. We note that one $U(1)$ rotation may induce several rotations in $\phi$ (but at least one) and if $\varepsilon$ parametrizes the $U(1)$ transformations, one has $\phi=n \varepsilon$ with $n$ integer. The Killing vector $K_{I}$ corresponding to this transformation is given by

$$
\begin{aligned}
& K_{1}^{\varphi}=n \\
& K_{1}^{\alpha}=0 \quad, \alpha \neq \varphi
\end{aligned}
$$

The $U(1)$ invariance of the ground state metric

$$
\delta_{v(1)} \dot{g}_{\alpha \beta}=-\varepsilon n \partial_{\varphi} \dot{g}_{\alpha \beta}=0
$$

implies that $\stackrel{\circ}{g}_{\alpha \beta}$ is $\phi$ independent. As well, we can always find a choice of $\stackrel{\circ}{\alpha}_{\alpha}^{a}$ which is $\phi$ independent. The U(1) invariance of $\stackrel{\circ}{\alpha}_{\alpha}^{a}$ implies that every $\phi$ dependence of $\stackrel{\circ}{\alpha}_{\alpha}^{a}$ is a pure Lorentz transformation and it can therefore be absorbed by a suitable chosen $\phi$ dependent Lorentz transformation, corresponding to a special choice of the veilbein basis. For this choice the function $\stackrel{\circ}{\varepsilon}_{1 \mathrm{ab}}$ [eq. (60)] vanishes identically. Supplementing the reflection $I_{\phi}(\phi \rightarrow-\phi)$ by a reflection of say, the first internal Lorentz index $I_{1}^{\ell}$ the internal veilbein components transform under $I_{1}^{\prime}=I_{\phi} I_{I}^{\ell}$ as

$$
\begin{aligned}
I_{1}: \quad & \hat{e}_{1}^{\varphi}\left(\varphi, y^{\alpha}, x\right) \rightarrow \hat{e}_{1}^{\varphi}\left(-\varphi, y^{\alpha}, x\right) \\
& \hat{e}_{1}^{\alpha}\left(\varphi, y^{\alpha}, x\right) \rightarrow-\hat{e}_{1}^{\alpha}\left(-\varphi, y^{\alpha}, x\right) \\
& \hat{e}_{a}^{\varphi}\left(\varphi, y^{\alpha}, x\right) \rightarrow-\hat{e}_{a}^{\phi}\left(-\varphi, y^{\alpha}, x\right) \\
& \hat{e}_{a}^{\alpha}\left(\varphi, y^{\alpha}, x\right) \rightarrow \hat{e}_{a}^{\alpha}\left(-\phi, y^{\alpha}, x\right), \alpha \neq \varphi, a \neq 1
\end{aligned}
$$


The ground state conserves the symmetry $I_{1}$ if $\stackrel{\circ \alpha}{e_{1}}=0, \stackrel{\circ \phi}{e}=0$ and correspondingly $I_{1}$ symmetry is broken only by non-zero non-diagonal elements $g_{\alpha \phi}$ of the metric. Next we have to investigate the action of $I_{1}$ on the generators of the gauge group. We split the generators is into the $\ell$ hermitian generators $s_{i}^{Q}$ of the Cartan-subalgebra ( $\ell$ is the rank of the group) and the remaining ladder operators $\mathrm{s}_{\mathrm{k}}^{ \pm}$. The commutation relations in this basis have the standard form:

$$
\begin{aligned}
& {\left[S_{i}^{Q}, S_{j}^{Q}\right]=0} \\
& {\left[S_{i}^{Q}, S_{i}^{+}\right]=\alpha_{i}^{(z)} S_{k}^{+},\left[S_{i}^{Q}, S_{z}^{-}\right]=-\alpha_{i}^{(k)} S_{z}^{-}}
\end{aligned}
$$

[The vectors $\left(\alpha_{1} \ldots \alpha_{\ell}\right)$ are called the roots of the algebra.] We take the generator is ${ }_{1}=$ in $\partial_{\phi}$ to be one of the generators of the Cartan subalgebra

$$
i S_{1}=i n \partial_{\phi}=S_{i_{0}}^{Q}
$$

Using coordinates on the orbit of the abelian group corresponding to the Cartan subalgebra, the other generators $s_{j}^{Q}, j \neq i_{0}$ can be written in the form

$$
S_{j}^{Q}=i\left(K_{j}^{\alpha} \partial_{\alpha}+K_{j}^{\varphi} \partial_{\varphi}\right), \alpha \neq \varphi, j \neq i_{0}
$$

where $\left[s_{i_{0}}^{Q}, s_{j}^{Q}\right]=0$ implies

$$
\partial_{\varphi} K_{j}{ }^{\alpha}=0, \quad \partial_{\varphi} K_{j}^{\varphi}=0
$$

Furthermore, we choose the $S_{i}^{Q}$ to be orthogonal with $k_{j}^{\phi}=0$. One immediately finds that $I_{1}$ reverses the sign of $S_{i_{0}}^{Q}$ and leaves all other $S_{j}^{Q}$ invariant:

$$
\begin{aligned}
& I_{1}^{-1} S_{i_{0}}^{Q} I_{1}=-S_{i_{0}}^{Q} \\
& I_{1}^{-1} S_{j}^{Q} I_{1}=S_{j}^{Q}, j \neq i_{0}
\end{aligned}
$$

Thus for any given system of weights, $I_{1}$ reverses the sign of the $i_{0}$ components of a11 weights.

We define the reflected generators

$$
\begin{aligned}
& \tilde{S}_{i}^{Q}=I_{1}^{-1} S_{i}^{Q} I_{1} \\
& \tilde{S}_{i}^{ \pm}=I_{1}^{-1} S_{i}^{ \pm} I_{1}
\end{aligned}
$$


If all reflected generators $\tilde{S}_{k}^{ \pm}$leave the ground state $K^{D}$ invariant, the reflection $I_{1}$ corresponds to an attomorphism of the algebra:

$$
\begin{aligned}
& \tilde{S}_{i_{0}}^{Q}=-S_{i_{0}}^{Q}, \quad \tilde{S}_{j}^{Q}=\tilde{S}_{j}^{Q}, j \neq i_{0} \\
& \tilde{S}_{\&}^{+}=a_{z \varepsilon^{\prime}}^{+} S_{q^{\prime}}^{+}+a_{z_{z^{\prime}}}^{-} S_{z^{\prime}}^{-}
\end{aligned}
$$

If $\mathrm{K}^{\mathrm{D}}$ is $\mathrm{I}_{1}$-invariant this is necessarily the case, but $I_{1}$ could be an automorphism even for spaces which axe not $I_{1}$ invariant. A necessary condition for I being an automorphism can be derived from the commutation relations:

$$
\begin{aligned}
& {\left[\tilde{S}_{i}^{a}, \tilde{S}_{z}^{ \pm}\right]= \pm \alpha_{i}^{(8)} \tilde{S}_{z}^{ \pm}} \\
& {\left[S_{i}^{a}, \tilde{S}_{i}^{ \pm}\right]= \pm \tilde{\alpha}_{i}^{(8)} \tilde{S}_{z}^{ \pm}}
\end{aligned}
$$

The vectors $\tilde{\alpha}_{i}^{(k)}$ are obtained from $\alpha_{i}^{(k)}$ by revexsing the sign of all $i_{0}$ components [compare eq. (72)]. If $I_{1}$ corresponds to an automorphism of the algebra, all $\tilde{\alpha}_{i}^{(k)}$ must again be roots of the algebra. The automorphism induced by $I_{I}$ is then a reflection of the root system on the $i_{0}$-axis.

An automorphism $I_{1}$ transforms any irreducible representation of the gauge group into an irreducible representation of the same dimension. In particular, if the spinors $\psi_{\mathrm{nH}}(\mathrm{y})$ are the components of an irreducible representation $\mathrm{H}$, then the reflected spinors

$$
\psi_{n H}^{I}\left(\varphi, y^{\alpha}\right)=I_{1} \psi_{n H}\left(\varphi, y^{\alpha}\right)=\eta_{a} \Gamma^{1}\left(\overline{\Gamma_{D}}\right) \psi_{n H}\left(-\phi, y^{\alpha}\right)
$$

are components of an irreducible representation of the same dimension:

$$
S_{z} \psi_{n H}^{I}=\psi_{n^{\prime} H}^{I}\left(T_{z}^{I}\right)_{n ' n} \text {. }
$$

For real or pseudoreal representations, the automorphism can be written in the form

$$
T_{z}^{I}=A T_{z} A^{-1}
$$

where the matrix A represents the reflection on the $i_{0}$ axis of the weight system. $I_{1}$ is called an inner automorphism if it can be represented as an element of the gauge group. In this case $I_{1}$ transforms every representation into itself. otherwise, $I_{1}$ is an outer automorphism which transforms complex representations into the complex conjugate ones or exchanges inequivalent real or pseudoreal representations with the same dimension or else transforms some representations into equivalent, but different representations. 
We can define similar coordinate reflections for the coordinates corresponding to the orbits of the other generators of the Cartan subalgebra. Consider the system of the resulting $\&$ coordinate reflections $I_{1}, I_{2}, \ldots I_{\ell}$. Every two of these reflections anticommute for spinors and commute when applied on tensors. Every product of these transformations is again a discrete transformation. In particular, we define the reflection of all coordinates corresponding to orbits of the Cartan subalgebra

$$
J_{e}=I_{1} I_{2} \ldots I_{e}
$$

The answer to the question which one of these discrete symmetries can correspond to an automorphism of the algebra is well known in mathematics (compare table 17 in ref. 9). The transformation $J_{\ell}$ can be an automorphism for all groups. It changes the sign of all weights and transforms $s_{k}^{+}$into $s_{k}^{-}$. $J_{\ell}$ transforms evexy complex representation into $i t s$ complex conjugate and every real or pseudoreal representation into itself. Thus $\mathrm{J}_{\ell}$ results in a four-dimensional transformation in the class $\&$. Reflection of only one coordinate cannot be an automorphism for the groups $G_{2}, E_{6}, E_{7}$, and $E_{8}$. The weight system obtained from the action of $I_{1}$ does not correspond to a representation of the group in this case. In particular, this implies that spaces admitting a symetry $G_{2}, E_{6}, E_{7}$, or $E_{8}$ cannot be symmetric with respect to a reflection of one coordinate. For SU(n) or $\mathrm{SO}(2 \mathrm{n}+1)$ the reflection $I_{1}$ can be an automorphism which transforms every representation into an equivalent representation (not necessarily the same). The four-dimensional symmetry belongs to the class $\mathcal{I}$. For $S 0(2 n)$ symmetric spaces $I_{1}$ exchanges inequivalent real or pseudoreal representations for $2 n=4 \bmod 4$ (it belongs to the class $\stackrel{i}{1})$ and transforms complex representations into complex conjugate ones for $2 n=2 \bmod 4$. In the second case $I_{1}$ corresponds again to a four-dimensional charge conjugation of the class $\stackrel{\circ}{\mathrm{C}}$. For $S O(n)$ symetric spaces all products of reflections $I_{i}$ can correspond to automorphisms of the algebra. An even number of reflections transforms all representations into equivalent ones. An odd number of reflections belongs always to the class $\stackrel{\complement}{C}$ for $\operatorname{So}(4 n+2)$ symmetric spaces. So $(n)$ symmetric internal spaces invariant with respect to an odd number of reflections 
are in fact $O(n)$ symmetric. We remember that an odd number of reflections is not contained in gen $_{D}$. Since the gauge group is a subgroup of gen $_{D}$, an odd number of reflections can only correspond to an outex automorphism. Conversely, an even number of reflections may be an inner automorphism and therefore harmless.

As a consequence, no internal space admitting a symmetry of the type $\mathrm{J}_{\ell}$ can yield a realistic theory since it induces a non-trivial conserved four-dimensional charge conjugation. Similarily, internal spaces admitting a symetry $I_{1}$ or an odd number of coordinate reflections of this type induce a non-trivial symmetry of the class $\tilde{I} \mathcal{I}$ or $\tilde{I} \tilde{C}$ in four dimensions which has not been observed in nature. If $I_{1}$ or $J_{\ell}$ are not symmetries of the action, the transformations $I_{1} P_{3}$ or $J_{\ell} P_{3}$ are and they are equally unacceptable. The vielbein and the metric of the ground state of a realistic Kalura-klein theory must break both $I_{1}$ and $J_{\ell} !$ There is, however, a difference between exceptional and orthogonal or unitary groups: the $I_{1}$ transform of an $E_{6}$ symmetric ground state metric is of course invariant under $E_{6}$ transformations corresponding to the generators $\tilde{S}$. However, it is not invariant with respect to the original $E_{6}$ transformations obtained by applying $S_{k}^{ \pm}$. On the contrary, if $I_{1}$ is an automorphism, the $I_{1}$ transform of an $S O(n)$ or SU( $n$ ) symetric metric is again invariant undex the transformation group constructed with the $S_{k}^{ \pm}$. Two different ground states with the same $S O(\mathfrak{n})$ symmetry may be transformed into each other by the action of $I_{1}$. Nevertheless, any given ground state breaks $I_{1}$ in this case. To resume, the internal space $K^{D}$ should not be invariant under an odd number of reflections of internal coordinates. This leads to four-dimensional symetries of the class $\tilde{\mathrm{P}}$ and $\tilde{\mathrm{T}}$. A symetry under an even number of internal coordinate reflections is only acceptable if this is a gauge transformation. Otherwise, unobserved non-trivial symmetries of the class ĨI or $\tilde{\mathrm{I}} \mathrm{C}$ are induced in four dimensions.

Let us look closer at the action of the reflections $I_{1} P_{3}, J_{\ell}$ (for $\ell$ even) or $\mathrm{J}_{\ell} \mathrm{P}_{3}$ (for $\ell$ odd) within the reduced four-dimensional theory. These transformations are always defined, even if the symmetry is broken by spontaneous compactification. We will assume from now on that $J_{\ell}$ and $I_{1}$ correspond to automorphisms of the algebra. One has 


\section{$I_{1} \psi_{n H \varepsilon}\left(\varphi_{1}, y^{\alpha}\right) \varphi_{n H \varepsilon}(x)=\eta_{a} \Gamma^{\alpha} \Gamma_{D} \psi_{n H \varepsilon}\left(-\varphi_{1} y^{\alpha}\right) \varphi_{n H \varepsilon}(x)$ \\ $=\psi_{n H \xi}\left(\varphi, y^{\alpha}\right) I_{n n^{\prime} H H^{\prime}+\xi^{\prime}} \varphi_{n^{\prime} H^{\prime} \xi^{\prime}}(x) \quad$ for $\mathrm{d}$ even}

\section{$I_{1} \psi_{n H \xi}\left(\varphi, y^{\alpha}\right) \varphi_{n H \xi}(x)=\eta_{a} \Gamma^{1} \psi_{n H \xi}\left(-\phi, y^{\alpha}\right) \bar{\gamma}^{5} \varphi_{n H \xi}(x)$ $=\psi_{n H z}\left(\varphi, y^{\alpha}\right) I_{n n^{\prime} H H^{\prime} \& \varepsilon^{\prime}} \bar{\gamma}^{5} \phi_{n^{\prime} H^{\prime} z^{\prime}}(x) \quad$ for d odd}

The constant coefficients $I_{\mathrm{nn}^{\prime} \mathrm{HH}^{\prime} \mathrm{kk}^{\prime}}$ characterize the four-dimensional action of $I_{1}$ and $I$ is proportional to $\delta_{\mathrm{HH}^{\prime}}$ if the automorphism carries every representation into an equivalent one.

We may characterize the $\psi_{n H k}$ as eigenvalues of the mass matrix $\psi_{n H k}=\psi_{n H M j}$

$$
\dot{\Gamma}^{\alpha} \dot{D}_{\alpha} \psi_{\text {nHmg }}(y)=M \psi_{n H \mu_{j}}(y)
$$

The mass operator $\stackrel{\circ}{\Gamma}^{\circ}{ }_{\alpha}^{\circ}$ is the Dirac operator on the internal manifold corresponding to the ground state vielbein $\stackrel{\circ}{\alpha}_{\alpha}^{a}$. Since $I_{1}$ (or $I_{1} P_{3}$ ) leaves the kinetic term of the higher dimensional spinor invariant, it commutes with $\Gamma^{\alpha} \mathrm{D}_{\alpha}$ for $\mathrm{D}$ even and anticommutes for $\mathrm{D}$ odd. If the ground state does not break $I_{1}$, these commutation relations can be transferred to the mass operator. The transformation $I_{1}$ carries every spinor into a spinor with equal mass ${ }^{2}$. For $D$ odd, the sign of the mass (which has no physical relevance) changes and $I_{1}$ necessarily transforms two different massive spinors into each other.

If the ground state is not $I_{1}$ invariant, one finds

$$
\left[I_{1}, r^{\alpha} \dot{D}_{\alpha}\right]=\left(\Gamma^{1} \Sigma^{a b} \dot{e}_{1 a b}+2 \Gamma^{a} \Sigma^{1 a} a_{a 1 a}-2 \Gamma^{1} e_{1}^{\alpha} \partial_{\alpha}-2 \Gamma^{a} e_{a}^{a} \dot{\xi}_{b}\right) I_{1}(83)
$$

with $a, b \neq 1$ and $\dot{\omega}$ the spin connection formed with the ground state vielbein. For $d$ odd, one has to replace the commutator on the left-hand side by an anticommutator and to multiply the right-hand side with a minus sign. Obviously, the right-hand side of eq. (83) vanishes for $\stackrel{\circ}{e}_{1}^{\alpha}=0, \stackrel{\circ}{\stackrel{\phi}{a}}=0$. If spontaneous compactification breaks $I_{1}$, this reflection transforms a spinor into a spinor of different mass. We note that the $I_{1}$ transform of a mass eigenstate is not 
necessarily an eigenstate of the mass operator again. Nevertheless, it would be interesting to investigate cases where mass eigenstates are transformed into each other and $I_{1}$ has some features of a ladder operator within the mass spectrum: of course, all the discussion can be applied similarily to the reflection $J_{\ell}$ and to other similar reflections.

To conclude this subsection, we show the strength of the constraints on acceptable internal spaces $\mathrm{K}^{\mathrm{D}}$ by illustrating the consequences of internal coordinate reflections on the possible existence of four-dimensional chiral fermions. Here we define chiral spinors with respect to the unification group. Any realistic theory must have such chiral spinors. [The existence of chiral spinors is connected with topological properties of $\mathrm{k}^{\mathrm{D}}{ }^{4}$ ).] We could imagine that the unified theory has a non-trivial $\overline{\mathrm{P}}, \overline{\mathrm{CP}}$, or $\overline{\mathrm{T}}$ invariance which could be spontaneously broken. Spontaneous breakdown of symmetries can equivalently be described by deformations of the internal space. Consider the space $\stackrel{\circ}{K}^{D}$ which is symetric under the unification group. $\stackrel{\circ}{K}^{D}$ is not the ground state, but it is related to the ground state $\mathrm{K}^{\mathrm{D}}$ by a continuous deformation. (This means that $\mathrm{K}^{\mathrm{D}}$ and $\mathrm{K}^{\mathrm{D}}$ have the same topology and differentiable structure.) Then we can perform dimensional reduction on $\mathrm{K}^{D}$ as well as on $\mathrm{K}^{\mathrm{D}}$ and the fact that $\stackrel{\circ}{\mathrm{K}}^{\mathrm{D}}$ is not the ground state will show up in the feature that the four-dimensional theory obtained from dimensional reduction on $k^{D}$ admits spontaneous symmetry breakdown. The vacuum expectation values of scalars reflect the difference in the metric of $k^{D}$ and $K^{D}$. Now we could imagine that $\stackrel{\circ}{K}$ has some non-trivial discrete symmetries. Of course, all non-trivial reflection symmetries of $\mathrm{K}^{\mathrm{D}}$ must finally be broken by the deformations to $\mathrm{K}^{\mathrm{D}}$. This corresponds to spontaneous breakdown of four-dimensional discrete symmetries. The restrictions on $\hat{\mathrm{K}}^{\mathrm{D}}$ from discrete symmetries could be less severe than the restrictions on the ground state $\mathrm{K}^{\mathrm{D}}$. However, $\stackrel{\circ}{\mathrm{K}}^{\mathrm{D}}$ should admit chiral four-dimensional spinors. Chiral fermions can never be produced by spontaneous symmetry breakdown ${ }^{4}$ and must exist in the unified theory. This will give us severe restrictions on possible reflection symmetries of ${ }_{\mathrm{K}}^{\mathrm{D}}$. 
We remember that $D$-dimensional versions of the symmetries $I_{1}$, $J_{\ell}$, etc. can always be defined and reflect themselves in the mass spectrum coming from the expansion of a D-dimensional Dirac spinor. Let us assume for a moment that $I_{\downarrow}$ and $J_{\ell}$ are symmetries of the internal space $\stackrel{\circ}{\mathrm{D}}$ corresponding to the unbroken unified theory. For $D$ even, we have the commutation relations

$$
\begin{aligned}
& \left\{I_{1}, \bar{\Gamma}_{D}\right\}=0 \\
& \zeta_{e} \bar{\Gamma}_{D}=(-1)^{\ell} \vec{\Gamma}_{D} J_{l}
\end{aligned}
$$

An odd number of reflections transforms a representation contained in $\psi^{+}(y)$ into a representation of the same dimension contained in $\psi^{-}(y)$. For d-dimensiona1 Wey1 spinors this corresponds to a flip in four-dimensional helicity. An even number of internal coordinate reflections does not change four-dimensional helicity. For $\ell$ even, $J_{\ell}$ is a non-trivial four-dimensional charge conjugation and chiral spinors are excluded. For $\ell$ odd, $\mathrm{P}_{3} \mathrm{~J}_{\ell}$ is a four-dimensional $C P$ conjugation which does not exclude chiral spinors. If $I_{1}$ is in the class $\stackrel{\circ}{\mathrm{C}}$, which is the case for a reflection of coordinates in the orbit of orthogonal groups $S O(4 n+2), P_{3} I_{1}$ is again a four-dimensional CP transformation consistent with chiral spinors. For all other cases $I_{1}$ is in the class $\dot{I}$ and the $\bar{P}$ transformation corresponding to $\mathrm{P}_{3} \mathrm{I}_{1}$ forbids the existence of chiral spinors for a ground state ${ }_{\mathrm{K}}^{\mathrm{D}}$. We note that D-dimensional charge conjugation also transforms all complex representations into complex conjugate ones, but may have different commutation properties with $\bar{\Gamma}_{D}$ compared to $J_{\ell}\left(C_{D} \vec{\Gamma}_{D}=(-1)^{D / 2} \bar{\Gamma}_{D} e_{D}\right)$. We illustrate the action of $J_{\ell}$ and $e_{D}$ on complex spinors graphically in fig. 1. Only fig. 1d is consistent with chiral spinors. Chiral fermions are consistent with an odd number of internal reflections only if such a transformation belongs to the class $\stackrel{\circ}{\mathrm{C}}$. This may be relevant if the symmetry group of $\mathrm{K}^{\mathrm{D}}$ is a direct product of SO(10) [or SU(5)] and a generation group $G_{F}$. If $G_{F}$ has only real or pseudoreal representations like so(3), SO(4), or SO(5), a reflection of one of the coordinates characterizing the orbit of $\mathrm{G}_{\mathrm{F}}$ carries a left-handed four-dimensional spinor into a right-handed one of the same representation. Chiral spinors are not consistent with such a reflection symmetry of $\stackrel{\circ}{\mathrm{K}}^{\mathrm{D}}$ : 


\section{SUPPLEMENTARY FIELDS IN HIGHER DIMENSIONS}

In this short section we discuss possible modifications of our investigation of discrete symmetries if one introduces supplementary fields in d dimensions. This is typically the case for higher dimensional supergravity theories. For example, supergravity in eleven dimensions needs an antisymmetric third rank tensor $\hat{A} \hat{\mu} \hat{\nu} \hat{\rho}$. The ground state is now characterized by vacuum expectation values of the metric and four-dimensional Lorentz scalars. We therefore can restrict our discussion to the effects of supplementary tensor fields in higher dimensions.

Two modifications of our previous discussion for pure gravity are possible: first, some of the d-dimensional discrete symmetries (compare table 2) may be violated by the couplings of d-dimensional tensors. An arbitrary tensor $\hat{A}_{\hat{\mu}} \hat{\nu}$... $\hat{\sigma}$ has the following transformation properties under symmetries of the type $T_{d}, P_{3}$, $I_{1}:$

$$
\begin{aligned}
& T_{\alpha} \hat{A} \hat{\mu} \hat{\imath} \ldots . . . \hat{\sigma}\left(x^{0}, x^{i}, \varphi, y^{\alpha}\right)=\varepsilon_{T} \hat{A}_{\hat{\mu}}^{T} \hat{v} \ldots \hat{\sigma}\left(-x^{0}, x^{i}, \varphi, y^{\alpha}\right) \\
& P_{3} \hat{A} \hat{\mu \nu} \ldots \hat{\sigma}\left(x^{0}, x^{i}, \varphi, y^{\alpha}\right)=\varepsilon_{P_{3}} \hat{A}^{P_{3}} \hat{\mu} \hat{\nu} . . . \hat{\sigma}\left(x^{0},-x^{i}, \varphi, y^{\alpha}\right) \\
& I_{1} \hat{A} \hat{\mu \nu} \ldots \hat{\sigma}\left(x^{0}, x^{i}, \phi, y^{\alpha}\right)=\varepsilon_{I_{1}} \hat{A}_{\hat{\mu}}^{I_{1}} \hat{\nu} \ldots \hat{\sigma}\left(x^{0}, x^{i},-\phi, y^{\alpha}\right)
\end{aligned}
$$

where $\hat{\mathrm{A}}^{\mathrm{T}}$ is obtained from $\hat{A}$ by multiplication with $(-1)^{\mathrm{J}_{0}}$ with $\mathrm{J}_{0}$ the number of indices taking the value zero, and similar rules for $\hat{A}^{P^{3}}$ and $\hat{A}^{I_{1}}$. A possible strategy is to determine the values $\varepsilon_{T}, \varepsilon_{P_{3}}$ and $\varepsilon_{I_{1}}$, which can take the values plus or minus one, from the coupling of the tensor to spinors and then to check if the action is invariant. Just as in four dimensions, scalars and pseudoscalars, for example, should not be mixed and couple to different fermion bilinears. We note that $I_{1}$ can be obtained from $P_{3}$ by a gend transformation fixing $\varepsilon_{I_{1}}=\varepsilon_{P_{3}}$. The transformation properties of some spinor bilinears under the symmetries $\mathrm{T}_{\mathrm{d}}$, $P_{3}, C_{d}$ and PCT are given in table 4 for arbitrary dimension $d$. With our phase conventions of Section 2 all bilinears of the type $\tilde{\psi} \Gamma{ }^{(\mathrm{k})} \psi$ with $\tilde{\psi}=\psi^{\mathrm{T}} \mathrm{C}_{\mathrm{d}}$ have the same transformation properties as bilinears of the type $\bar{\psi} \Gamma^{(\mathrm{k})} \psi$. 
Similar as in four dimensions, the symmetry $\mathrm{P}_{d} e_{d} \mathrm{~T}_{d}$ is always a symmetry of the action for $\mathrm{d}$ even. For Majorana spinors $\mathcal{C}_{\mathrm{d}}$ is trivial and $\mathrm{P}_{\mathrm{d}} \mathrm{e}_{\mathrm{d}} \mathrm{T}_{\mathrm{d}}$ reduces to a $P_{d} T_{d}$ symmetry. The symmetry $P_{3} T_{d}$ is then obtained by a gen ${ }_{d}$ transformation and is again a symmetry of the action. It corresponds to four-dimensional $\overline{\mathrm{PC}} \overline{\mathrm{T}} \mathrm{sym}-$ metry. It cannot be broken by spontaneous compactification. For odd dimensions, $\mathrm{P}_{3} \mathrm{C}_{\mathrm{d}} \mathrm{T}$ is always a symmetry of the action (this is not true for $\mathrm{P}_{\mathrm{d}} \mathrm{e}_{\mathrm{d}} \mathrm{T}_{\mathrm{d}}$ ) and yields the usual four-dimensional $\overline{\mathrm{PCT}}$ transformation if $\mathfrak{C}_{\mathrm{d}}$ is trivial.

A second modification is related to further possibilities of breakdown of discrete symmetries by spontaneous compactification. Four-dimensional pseudoscalars may acquire vacuum expectation values and break $\bar{P}$. For example, there could be vacuum expectation values of some antisymetric four-index tensor proportional to the totally antisymmetric four-dimensional $\varepsilon$-tensox. Such an expectation value is Lorentz invariant but breaks $\overline{\mathrm{T}}$ and $\overline{\mathrm{P}}$ invariance. Actually, the action of eleven dimensional supergravity is $\mathrm{T}_{\mathrm{d}}$ invariant. The corresponding four-dimensional $\overline{\mathrm{CT}}$ invariance can only be broken by a non-zexo vacuum expectation value of the Minkowski components of the field strength corresponding to the three-index tensor $\widehat{A}_{\hat{\mu}} \hat{v} \hat{p}$ :

$$
\begin{aligned}
& \hat{F}_{\hat{\mu} \hat{\nu} \hat{\rho} \tilde{\sigma}}=D_{[\hat{\mu}} \hat{A} \hat{\gamma \rho \hat{\sigma} \sigma]} \\
& \left\langle\hat{F}_{\mu \nu \rho \sigma}\right\rangle \sim \varepsilon \operatorname{\varepsilon p\rho \sigma }
\end{aligned}
$$

which characterizes certain solutions ${ }^{6}$ ) of the field equations.

\section{CONCLUSIONS AND DISCUSSION}

We have seen that discrete symmetries put many constraints on possible realistic Kaluza-Klein theories. The first class of constraints limits the possible $\mathrm{d}$-dimensional theories to start with. These come from symetries like d-dimensional time reversal, reflection of an odd number of coordinates and charge conjugation. $\mathrm{T}_{\mathrm{d}}, \mathrm{P}_{3}$, and $\mathrm{C}_{\mathrm{d}}$ cannot be broken by the ground state of pure gravity theories. Therefore theories of pure higher dimensional gravity should not be invariant under $T_{d}, P_{3}, e_{d} P_{3}$, or a non-trivial $e_{d}$. Otherwise, unobserved discrete symmetries are induced in four dimensions. This limits the possible theories of pure gravity coupled to a spinor to the case of a Majorana-Weyl spinor in $\mathrm{d}=2$ mod 8 dimensions. 
The constraints on the d-dimensional theory become less stringent if supplementary fields are introduced. This can allow the breaking of $\mathrm{T}_{\mathrm{d}}$ and $\mathrm{P}_{3}$ invariance in the purely bosonic part of the effective d-dimensional action. If this is not the case, like in $d=11$ supergravity which has a $T_{d}$ and $P_{3}$ invariance of the action, there is still a way out by breaking $T_{d}$ and $P_{3}$ through spontaneous compactification. This requires a totally antisymmetric tensor of rank four or the field strength of a totally antisymmetric tensor of rank three to acquire a vacuum expectation value $\sim \varepsilon_{\mu \nu \rho \sigma}$ for its Minkowski indices. This is exactly what happens for some solutions of spontaneous compactification in $\mathrm{d}=11$ supergravity and parity and CT are therefore broken without a d-dimensional Weyl constraint.

For the derivation of these results we have assumed that the pure gravitational part of the d-dimensional action (omitting the spinors) is invariant under time reversal and reflection of an odd number of spacelike coordinates. This requires that the function $\mathrm{f}\left(\hat{\mathrm{g}}_{\hat{\mu}} \hat{v}\right)$ in the effective action (including gen $\mathrm{d}$ invariant contributions from quantum fluctuations) contains only scalars but no pseudoscalars. However, in four dimensions a pseudoscalar can be constructed from the metric tensor and its derivatives:

$$
R_{\mu \nu \sigma \lambda} \tilde{R} \mu \nu \sigma \lambda=\frac{1}{2} R_{\mu \nu \sigma \lambda} R_{\rho \tau} \sigma^{\sigma \lambda} \varepsilon^{\mu \nu \rho \tau}(-g)^{-\frac{1}{2}}
$$

It violates $\overline{\mathrm{P}}, \overline{\mathrm{CP}}$, and $\overline{\mathrm{T}}$. Its space time integral corresponds to a topological charge, the Pontryagin number

$$
P=\frac{1}{16 \pi^{2}} \int d^{4} \times(-g)^{1 / 2} R_{\mu \nu \sigma \lambda} \tilde{R} \mu r \sigma \lambda
$$

This is very similar to a possible $\overline{\mathrm{CP}}$ violating pseudoscalar contribution to the effective action from gauge fields

$$
\theta F_{\mu \nu} \tilde{F}_{\mu \nu}^{\mu \nu}=\theta F_{\mu \nu} F_{\rho \sigma} \varepsilon^{\mu \nu \rho \sigma}(-g)^{-\frac{1}{2}}
$$

which is at the origin of the strong CP problem. Similarily, a term $\varepsilon R_{\mu \nu \sigma \lambda} \tilde{R}^{\mu \nu \sigma \lambda}$ in the effective action in four dimensions gives rise to a gravitational $\mathrm{CP}$ violation. 
We do not know in which dimensions a pseudoscalar can be constructed from the metric and its derivatives. It would require a non-vanishing contraction of products of the curvature tensor and Ricci tensor and their covariant derivatives with an odd number of totally antisymmetric d-dimensional $\varepsilon$-tensors. The generalized construction

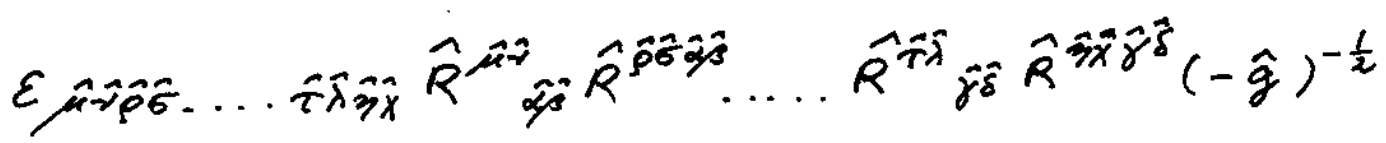

is only possible in $\mathrm{d}=4 \mathrm{mod} 4$ dimensions. The absence of a gravitational pseudoscalar in two dimensions suggests that gxavitational pseudoscalars could be absent for $d=2 \bmod 4$-- this remains to be shown (or found in the mathematical literature). Even if gravitational pseudoscalars are possible, they involve many derivatives if the number of dimensions is sufficiently high and their contribution to the reduced four-dimensional theory may be very small.

The question of gravitational pseudoscalars in higher dimensions is strongly related to the strong $C P$ problem in four dimensions. Suppose that there exists no such gxavitational pseudoscalar in d dimensions. The gen invariant part of the effective action without spinors is then $\mathrm{T}_{\mathrm{d}}$ and $\mathrm{P}_{3}$ invariant. If the ground state is a direct product of Minkowski space and an internal space $\mathrm{K}^{\mathrm{D}}$ these symmetries cannot be broken by spontaneous compactification in purely gravitational theories. The purely bosonic part of the effective action in four dimensions

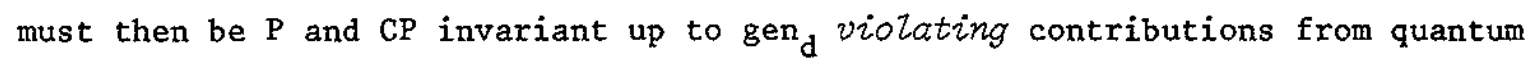
fluctuations involving length scales larger than the scale of spontaneous compactification. No $\mathrm{F}_{\mu \nu} \tilde{\mathrm{F}}^{\mu \nu}$ term can be obtained from dimensional reduction in this case. This means that the $\theta$ parameter vanishes at the scale of spontaneous compactification. A non-zero value at present energies can only be induced by quantum fluctuations, and it must be due to the $\mathrm{CP}$ violation in the fermionic sector. This is calculable and turns out ${ }^{10}$ ) to be typically very small. We also have to check that the determinant of the quark mass matrix obtained from dimensional reduction is real. This can be done by applying the general results of ref. 3 to the case where the isometry group of the ground state is the unbroken gauge group $\mathrm{SU}(3)_{\mathrm{C}} \times \mathrm{U}(1)_{\mathrm{em}}$. It follows that the quark mass matrix is hermitean and the determinant is therefore real. CP violation in the fermionic sector occurs at most 
in weak interactions, but not through the couplings to the massless fields. As a consequence, no strong $C P$ problem exists in those theories of pure gravity coupled to spinors which either do not admit gravitational pseudoscalars at all or where the contribution of such pseudoscalars to the effective action in four dimensions is sufficiently small. Kaluza-Klein theories may be an elegant new way to get rid of one of our long-standing problems:

The second class of constraints obtained from discrete symmetries concerns the structure of the internal space $\mathrm{K}^{\mathrm{D}}$. Non-trivial symmetries from reflections of internal coordinates (which cannot be obtained by gauge transformations) lead to unacceptable discrete symmetries in four dimensions. Some of these internal reflections lead to four-dimensional charge conjugation. As has been noted before, this gives a simple physical (geometrical) interpretation to the four-dimensional charge conjugation. The internal space $\mathrm{K}^{\mathrm{D}}$ of the ground state of a realistic Kaluza-Klein theory should not have any non-trivial reflection synmetry:

Reflection symetries of $\mathrm{K}^{\mathrm{D}}$ and of the space $\stackrel{\circ}{\mathrm{K}}^{\mathrm{D}}$ corresponding to the unbroken unified gauge symmetry are closely related to the possible existence of chiral spinors. Chiral spinors are perhaps the most serious problem for realistic Kaluza-Klein theories at this time. Possible candidates of theories admitting chiral spinors get more and more restricted -- which gives some hope of finding them if they exist.

Pure considerations of the Clifford algebra in $\mathrm{d}$ and $\mathrm{D}$ dimensions restrict D to be $2 \bmod 4$ for all theories where the gauge group is a subgroup of gen ${ }^{3}$ ). [This is the case for the typical Kaluza-Klein theories where the gauge group is related to isometries of the metric of $\mathrm{K}^{\mathrm{D}}$. On the contrary, hidden local symmetries like $\mathrm{SU}(8)$ in $\mathrm{d}=11$ supergravity are not of this type.] Indeed, a Weyl constraint is needed in d dimensions in order to make possible a difference between left-handed and right-handed spinors in four dimensions. This difference is related to possible differences in the mass and representation spectrum of the inequivalent D dimensional Weyl spinors. Chiral four-dimensional fermions require the D dimensional Weyl spinors to belong to complex representations of the Ddimensional Lorentz group SO(D). This restricts D to 2 mod 4. 
The existence of a non-trivial d-dimensional charge conjugation leads to an unwanted doubling of the four-dimensional mass spectrum for fermions. $e_{d}$ cannot be broken by any ground state of pure gravity. This requires d-dimensional Majorana spinors and restricts $D$ to be $6 \bmod 8$ for realistic theories.

Due to a theorem by Lichnerowicz ${ }^{12}$ ) we know that no massless spinors exist if the curvature scalar of $\mathrm{K}^{\mathrm{D}}$ is negative or zero everywhere on $\mathrm{K}^{\mathrm{D}}$ but not identically zero (in conventions where the curvature scalar for spheres is negative). Doing dimensional reduction by steps ${ }^{3}$ ) this can be generalized to a space $\mathrm{K}^{\mathrm{D}}$ which is a direct product of several internal spaces $k^{D}=k_{1} \times k_{2} \times \ldots \times k_{n}$. None of the spaces $K_{i}$ should have negative scalar curvature in order for $K^{D}$ to admit chiral fermions. The argument can be extended to the case where one of the $\mathrm{K}_{i}$ is a one dimensional circle $\mathrm{S}^{1}$. Starting with an even dimensional theory and first reducing to a d-1 dimensional theory be integrating out the $\mathrm{s}^{1}$ coordinate leaves us with a massless fermion in d-1 dimensions. However, this fermion is neutral with respect to the U(1) group of the circle. Further dimensional reduction to an effective four dimensional theory cannot give chiral fermions since we now start with a vector-like theory in odd dimensions. An internal space $K^{D}=K^{D-1} \times S^{1}$ admits no chiral fermions.

These arguments become very powerful constraints when combined with topological considerations ${ }^{4)}$. The number of chiral fermions is not changed by continuous deformations of $\mathrm{K}^{\mathrm{D}}$ leaving the gauge group invariant. Chiral fermions must be in complex representations with respect to at least one $U(1)$ subgroup of the isometries of $\stackrel{\circ}{\mathrm{K}}^{\mathrm{D}}$. (In a realistic theory this $U(1)$ subgroup can be identified with hypexcharge.) This requires an internal space $\stackrel{\circ}{\mathrm{K}}^{\mathrm{D}}$ which cannot be changed continuously, while keeping the $U(1)$ subgroup unbroken, into one of the spaces discussed in the precedent paragraph. Especially, the U(1) group must have fixpoints [points in $\mathrm{K}^{\mathrm{D}}$ where the orbit of this $\mathrm{U}(1)$ is shrunk to a point]. Otherwise, $\mathrm{K}^{\mathrm{D}}$ has topology $\mathrm{K}^{\mathrm{D}-1} \times \mathrm{s}^{1}$ and does not admit chiral fermions. The number or chiral fermions becomes to a large extent a property of the topology and differentiable structure of $\mathrm{K}^{\mathrm{D}}$ : 
As we have shown in this paper, another class of constraints is related to possible discrete symetries of $\stackrel{\circ}{K}^{D}$. Chiral fermions are excluded if $\stackrel{\circ}{K}$ admits a symmetry of one of the following two types

i) an odd number of coordinate reflections corresponding to an automoxphism of the algebra which does not transform complex representations of the gauge group into complex conjugate ones;

ii) an even number of coordinate reflections which corresponds to an automorphism exchanging complex representations with their conjugates. Combined with index considexations, this argument is again very restrictive. In realistic gauge theories there are often vector-like $U(1)$ subgroups commuting with the chiral $U(1)$ subgroup. [In the standard mode1, the thixd component of weak isospin generates always a vector-like $U(1)$ group since $\mathrm{SU}(2)$ has not complex representations and it commutes with the chiral hypercharge generator.] If $\mathrm{o}^{D}$ has a reflection symmetry with respect to the coordinate $\Phi_{\mathrm{v}}$ parametrizing the orbit of the vector-like $U(1)$ group, it cannot exhibit chiral fermions.

The metric of any internal space which is invariant under both a vector-like and a commuting "chiral" $\mathrm{U}(1)$ group must be independent of both $\phi_{\mathrm{V}}$ and $\phi_{c}{ }_{c} \phi_{c}$ is the coordinate parametrizing the orbit of the "chiral" U(1) group]. As discussed in section 4 , breaking of the discrete symmetry $\Phi_{v} \rightarrow-\phi_{v}$ is then only possible by non-diagonal terms in the metric. However, if it is possible to make these nondiagonal terms vanish be a continuous deformation not breaking the "chiral" U(I), then such a space does again not admit chiral fermions. In pure gravity coupled to spinors it seems to be rather difficult to avoid this possibility and to construct spaces which actually admit chiral fermions.

\section{Note added}

After completing this work, I learned from E. Witten that there exists a proof by Atiyah and Hirzebruch ${ }^{12)}$ that the relevant index counting the number of chiral zero modes of the Dirac operator on the internal space vanishes for alZ compact 
internal spaces with a positive (or negative) definite signature of the metric. Thus, no chiral fermions can be obtained from dimensional reduction of standard gravity in higher dimensions coupled to a generalized spin $\frac{1}{2}$ fermion, if the ground state has the usual direct product form $\mathrm{M}^{4} \times \mathrm{K}^{\mathrm{D}}$.

I am very grateful to E. Witten for his hospitality in Princeton and for explaining me his own proof of the above theorem. 


\section{REFERENCES}

1) Th. Kaluza, Sitzungsber. Preuss. Akad. Wiss. Berlin, Math. Phys. K1, 966 (1921).

0. K1ein, Z. Phys. 37,895 (1926). .

J. Rayski, Acta Physica Polonica 27, 89 (1965).

R. Kerner, Ann. Inst. Henri Poincaré 9, 143 (1968).

Y.M. Cho and P.G.0. Freund, Phys. Rev. D 12,1711 (1975).

J.F. Luciani, Nuc1. Phys. B135, 111 (1978).

J. Scherk and J.H. Schwarz, Nuc1. Phys. B153, 61 (1979).

E. Witten, Nuc1. Phys. B186, 412 (1981).

A. Salam and J. Strathdee, Ann. Phys. 141, 316 (1982).

C. Wetterich, Phys. Lett. $110 \mathrm{~B}, 379$ (1982).

2) W. Thirring, Acta Physica Austriaca, Supp1. IX, 256 (1972).

P.G.0. Freund, Chicago preprint EFI 81/83 (1983).

3) C. Wetterich, Nuc1. Phys. B222, 20 (1983).

4) C. Wetterich, Nuc1. Phys. B223, 109 (1983).

5) E. Cremmer and B. Julia, Nucl. Phys. B159, 141 (1979).

6) P.G.0. Freund and M.A. Rubin, Phys. Lett. 97B, 233 (1980).

M.J. Duff, Nuc1. Phys. B219, 389 (1983).

7) C. Wetterich, Phys. Lett. 113B, 377 (1982).

8) C. Wetterich, Nucl. Phys. B211, 177 (1983).

9) R. Slansky, Group Theory for unified model building, Phys. Rep. C, 79; I (1981).

10) J. E1lis and M.K. Gaillard, Nuc1. Phys. B150, 141 (1979).

11) A. Lichnerowicz, C.R. Acad. Sci. Paris, Sêrie A-B 257, 7 (1963).

12) M.F. Atiyah and F. Hirzebruch, in Essays on topology and related topics, p. 18, ed. A. Haefliger and R. Narasimhan, Springer, Berlin, 1970. 
$\underline{\text { Table } 1}$

Discrete transformations of spinors in arbitrary dimensions d $\psi(x) \rightarrow U \psi\left(x^{\prime}\right)$ or $\psi(x) \rightarrow U \psi^{*}\left(x^{\prime}\right):$ complex conjugation is symbolized by (*)

\begin{tabular}{|c|c|c|c|c|c|c|}
\hline $\mathrm{d}\left[\begin{array}{ll}\bmod & 8\end{array}\right]$ & $P_{d}$ & $e_{d}$ & $\mathrm{~T}_{\mathrm{d}}$ & ${ }^{(\mathrm{PCT})} \mathrm{d}$ & $\mathrm{I}_{\mathrm{a}}$ & $\mathrm{P}_{3}$ \\
\hline 2 & $i \gamma^{0}$ & $\mathrm{~B}_{1}^{-1}(*)$ & $-i \gamma^{0} \bar{\gamma} B_{1}^{-1}(*)$ & $\bar{\gamma}$ & $i \gamma^{a} \bar{\gamma}$ & $\gamma^{0} \bar{\Gamma}_{\mathrm{D}}$ \\
\hline 3 & $i \gamma^{0}$ & $B_{1}^{-1}(*)$ & $-i \gamma^{0} B_{1}^{-1}(*)$ & 1 & $i \gamma^{a}$ & $-\gamma^{0} \bar{\gamma}^{5}$ \\
\hline 4 & $i \gamma^{0}$ & $\mathrm{~B}_{1}^{-1}(*)$ & $\gamma^{0} \bar{\gamma} B_{I}^{-1}(*)$ & $i \bar{\gamma}$ & $\gamma^{\mathrm{a}} \bar{\gamma}$ & $i \gamma^{0} \overline{\mathrm{T}}_{\mathrm{D}}$ \\
\hline 5 & $\gamma^{0}$ & $\mathrm{~B}_{2}^{-1}(*)$ & $\gamma^{0} B_{2}^{-1}(*)$ & -1 & $\gamma^{a}$ & $-i \gamma^{0} \bar{\gamma}^{5}$ \\
\hline 6 & $\gamma^{0}$ & $\mathrm{~B}_{2}^{-1}(*)$ & $\gamma^{0} \bar{\gamma} B_{2}^{-1}(*)$ & $-\bar{\gamma}$ & $\gamma^{\mathrm{a}} \bar{\gamma}$ & $i \gamma^{0} \tilde{\Gamma}_{D}$ \\
\hline 7 & $i \gamma^{0}$ & $\mathrm{~B}_{1}^{-1}(*)$ & $-i \gamma^{0} B_{1}^{-1}(*)$ & -1 & $i \gamma^{a}$ & $-\gamma^{0} \bar{\gamma}^{5}$ \\
\hline 8 & $\gamma^{0}$ & $\mathrm{~B}_{2}^{-1}(*)$ & $-i \gamma^{0} \bar{\gamma} B_{2}^{-1}(*)$ & $-i \bar{\gamma}$ & $i \gamma^{a} \bar{\gamma}$ & $\gamma^{0} \bar{\Gamma}_{D}$ \\
\hline 9 & $\gamma^{0}$ & $\mathrm{~B}_{2}^{-1}(*)$ & $\gamma^{0} B_{2}^{-1}(*)$ & 1 & $\gamma^{a}$ & $-i \gamma^{0} \tilde{\gamma}^{5}$ \\
\hline
\end{tabular}

$\underline{\text { Table } 2}$

Possible discrete symmetries consistent with Majorana and/or Weyl constraints

\begin{tabular}{|c|c|l|}
\hline$d[\bmod 8]$ & Type of spinor & Discrete symetries \\
\hline 2 & $\mathrm{M}-\mathrm{W}$ & $\mathrm{PT}$ \\
3 & $\mathrm{M}$ & $\mathrm{P}, \mathrm{T}, \mathrm{I}_{\mathrm{a}}, \mathrm{P}_{3}$ \\
4 & $\mathrm{~W}$ & $\mathrm{CP}, \mathrm{T}, \mathrm{CI}, \mathrm{CP}_{3}$ \\
& $\mathrm{M}, \mathrm{T}, \mathrm{I}_{\mathrm{a}}, \mathrm{P}_{3}$ \\
5 & $\mathrm{D}$ & $\mathrm{C}, \mathrm{P}, \mathrm{T}, \mathrm{I}_{\mathrm{a}}, \mathrm{P}_{3}$ \\
6 & $\mathrm{~W}$ & $\mathrm{C}, \mathrm{PT}$ \\
7 & $\mathrm{D}$ & $\mathrm{C}, \mathrm{P}, \mathrm{T}, \mathrm{I}_{\mathrm{a}}, \mathrm{P}_{3}$ \\
8 & $\mathrm{~W}$ & $\mathrm{CP}, \mathrm{T}, \mathrm{CI}, \mathrm{CP}_{3}$ \\
& $\mathrm{M}$ & $\mathrm{P}, \mathrm{T}, \mathrm{I}_{\mathrm{a}}, \mathrm{P}_{3}$ \\
\hline
\end{tabular}

D: Dirac spinor, M: Majorana spinor, W: Weyl spinor, M-W: Majorana-Wey1 spinor. 


\section{Table 3}

Some elements of the four-dimensional symmetry classes $\tilde{\mathrm{I}}, \tilde{\mathrm{P}}, \tilde{\mathrm{T}}$, and $\tilde{\mathrm{PT}}$

$$
\begin{array}{ll}
\tilde{I}: & \left(1, e_{d}, I_{a}, \Gamma, \bar{C}, \ldots\right) \\
\tilde{P}: & \left(P_{3}, e_{d} P_{3}, I_{x}, P_{3} I_{a}, P_{d}, \bar{P}, \gamma^{5} \bar{P} \ldots\right) \\
\tilde{T}: & \left(T_{d}, e_{d} T_{d}, I_{a} T_{d}, \bar{T}, \overline{C T}, \ldots\right) \\
\tilde{P T}: & \left(P_{3} T_{d}, P_{d} T_{d},(P C T)_{d}, \overline{P T}, \overline{P C T}, \ldots\right)
\end{array}
$$

\section{Table 4}

\begin{tabular}{|c|c|c|c|c|}
\hline Bilinear & $\varepsilon_{\mathrm{P}}$ & $\varepsilon_{T}$ & $\varepsilon_{e}$ & $\varepsilon_{\mathrm{PeT}}$ \\
\hline $\bar{\psi} \psi$ & 1 & $(-1)^{d+1} \alpha_{B}$ & $-\alpha_{B}$ & $(-1)^{\mathrm{d}}$ \\
\hline $\bar{\psi} \gamma^{\hat{m}_{\psi}} \psi$ & 1 & -1 & -1 & 1 \\
\hline $\bar{\psi} \Sigma^{\hat{m}} \hat{n}_{\psi}$ & 1 & $(-1)^{d} \alpha_{B}$ & $\alpha_{B}$ & $(-1)^{d}$ \\
\hline $\left.\bar{\psi} \gamma^{[\hat{x}} \hat{n} \hat{n}\right]_{\psi}$ & 1 & 1 & 1 & 1 \\
\hline $\bar{\psi} \bar{\gamma} \psi$ & -1 & $(-1)^{d / 2} \alpha_{B}$ & $(-1)^{(d-2) / 2} \alpha_{B}$ & 1 \\
\hline $\bar{\psi} \gamma^{\hat{\mathrm{m}}} \bar{\gamma} \psi$ & -1 & $(-1)^{(d-2) / 2}$ & $(-1)^{d / 2}$ & 1 \\
\hline
\end{tabular}

Transformation properties of fermion bilinears under discrete transformations in arbitrary dimensions (for example $e_{d}: \bar{\psi}_{\gamma} \hat{\mathrm{m}}_{\psi} \rightarrow \varepsilon_{\mathfrak{e}} \bar{\Psi}^{\hat{\mathrm{m}}_{\psi}}=-\bar{\psi}_{\gamma} \hat{\mathrm{m}}_{\psi}$ )

$\alpha_{B}=\left\{\begin{array}{l}-1 \text { for } B_{d}=B_{1} \\ +1 \text { for } B_{d}=B_{2}\end{array}\right.$ 


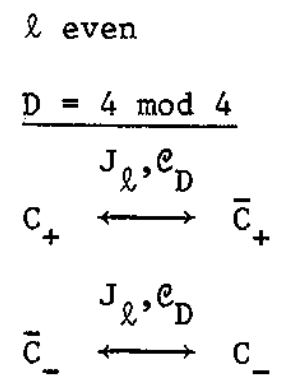

Fig. la

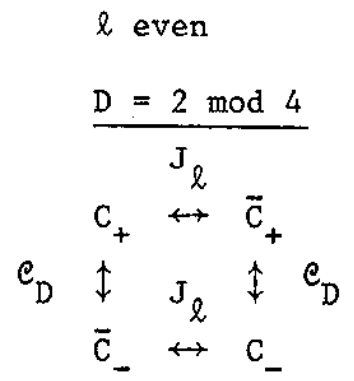

Fig. Tb

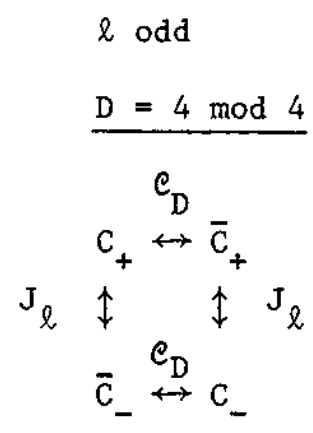

Fig. 1c

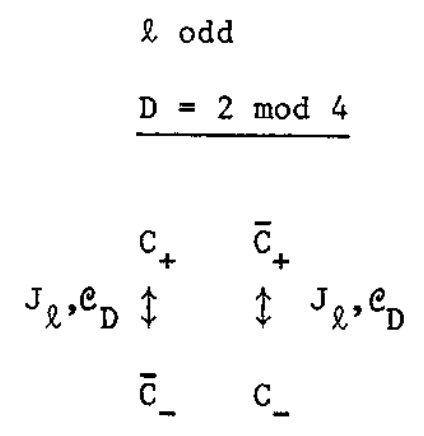

Fig. Id

Fig. 1 Action of $e_{D}$ and $J_{\ell}$ on $D$-dimensional WeyI spinors belonging to a complex representation of the isometry group. $C_{+}$denotes a complex representation in $\psi^{+}(\mathrm{y})$. 